\title{
Temperature-dependent oscillation modes in rotating superfluid neutron stars
}

\author{
V. A. Dommes, E. M. Kantor, M. E. Gusakov \\ Ioffe Institute, Polytekhnicheskaya 26, 194021 St. Petersburg, Russia
}

17 August 2021

\begin{abstract}
We calculate the spectrum of inertial oscillation modes in a slowly rotating superfluid neutron star, including, for the first time, both the effects of finite temperatures and entrainment between superfluid neutrons and protons. We work in the Newtonian limit and assume minimal core composition (neutrons, protons and electrons). We also developed an approximate method that allows one to calculate the superfluid r-mode analytically. Finally, we derive and analyze dispersion relations for inertial modes in the superfluid NS matter in the short wavelength limit.
\end{abstract}

Key words: stars: neutron - stars: oscillations (including pulsations) - stars: rotation

\section{INTRODUCTION}

According to the standard r-mode theory, hot and rapidly rotating neutron stars (NSs) in low-mass X-ray binaries (LMXBs) should be CFS-unstable with respect to emission of gravitational waves (Andersson 1998; Friedman \& Morsink 1998). As a consequence, the probability of observing them should be very small, but this conclusion contradicts observations (Ho et al. 2011). A number of possible ideas have been proposed to solve the paradox (see, e.g. Andersson \& Kokkotas 2001; Haskell et al. 2012; Ho et al. 2011; Mahmoodifar \& Strohmayer 2013), but its complete resolution is still lacking. Gusakov et al. (2014a,b) introduced a new scenario, in which the finite-temperature effects in the superfluid core of an NS lead to resonance coupling and enhanced damping (and hence stability) of oscillation modes at certain "resonance" stellar temperatures. It was demonstrated that NSs in LMXBs with high spin frequency may spend a substantial amount of time at these resonance temperatures, so that their interpretation does not constitute a problem.

The proposed resonance stabilization scenario was based on a simplified phenomenological model (in particular, resonance temperatures have never been explicitly calculated). To put it on a more solid ground, one has to calculate spectra of rotational inertial modes (modes for which the restoring force is the Coriolis force) for realistic superfluid NS models at arbitrary temperatures in order to find resonance temperatures at which the normal $r$-mode exhibits an avoided crossing with another mode. However, in most works (e.g. Lindblom \& Mendell 2000; Prix et al. 2002; Lee \& Yoshida 2003; Andersson et al. 2009) the inertial modes are studied only in zero-temperature limit, when all neutrons and protons are assumed to be in a superfluid state.

Kantor \& Gusakov (2017) considered normal and superfluid r-modes, incorporating finite-temperature effects and stratification by muons $(\mu)$, and subsequently found avoided crossings between normal and superfluid $r$-modes in the next-to-leading order in stellar rotation frequency. This work ignored the entrainment between superfluid neutrons and protons, which significantly affects the spectrum of superfluid inertial modes (see, e.g. Lee \& Yoshida 2003). Also Kantor \& Gusakov (2017) focused only on $r$-modes and have not studied other inertial modes, which also can interact with the normal $r$-mode. To fill this gap, we calculate the spectrum of inertial modes in superfluid NSs whose cores consist of neutrons $(n)$, protons $(p)$ and electrons $(e)$, accounting for both entrainment and finite-temperature effects. In Section 2 we provide the equations governing these oscillation modes, and in Section 3 we discuss the general classification of inertial modes. Section 4 presents the results of numerical calculations for inertial modes, obtained under assumptions of npe NS core composition and constant critical temperatures. In Section 5 we also present an approximate method that allows for calculation of the superfluid $r$-mode analytically, in the limit of small entrainment. In Section 6 we derive dispersion relations for inertial modes in superfluid npe matter in short-wavelength limit, and explain some properties of these modes that can be observed in the numerical results of Section 4. Finally, we provide a summary in Section 7 .

(C) 0000 The Authors 


\section{EQUATIONS GOVERNING OSCILLATIONS OF A ROTATING SUPERFLUID NS}

In this section we describe oscillations of superfluid NS using the Newtonian limit of relativistic hydrodynamics, formulated by Gusakov (2016); Gusakov \& Dommes (2016). We consider a slowly rotating (with the spin frequency $\Omega$ ) NS with the core composed of neutrons, protons and electrons. Possible superfluidity of baryons (neutrons and protons) is encoded in the symmetric entrainment matrix $Y_{i k}$ (Gusakov \& Andersson 2006; Gusakov et al. 2009a,b, 2014c) which is the relativistic analogue of the superfluid mass-density matrix $\rho_{i k}$ (Andreev \& Bashkin 1976). This matrix enters the neutron $(i=\mathrm{n})$ and proton $(i=\mathrm{p})$ four-current density $j_{(i)}^{\mu}$ :

$j_{(i)}^{\mu}=n_{i} u^{\mu}+Y_{i k} w_{(k)}^{\mu}$.

Here and hereafter indices $i, k$ run over neutrons and protons $(i, k=\mathrm{n}, \mathrm{p})$, and we assume summation over repeated indices. $u^{\mu}$ is the four-velocity of the non-superfluid component (electrons as well as baryonic thermal excitations), and the four-vector $w_{(k)}^{\mu}$ is expressed through the superfluid velocity $v_{(s k)}^{\mu}$, relativistic chemical potential $\mu_{k}$ and bare mass $m_{k}$ of particle species $k$ as $w_{(k)}^{\mu}=m_{k} v_{(s k)}^{\mu}-\mu_{k} u^{\mu}$. Electron four-current is $j_{(\mathrm{e})}^{\mu}=n_{\mathrm{e}} u^{\mu}$. From the condition $j_{(\mathrm{e})}^{\mu}=j_{(\mathrm{p})}^{\mu}$, which is valid for low-frequency hydrodynamic oscillations (Mendell 1991), it follows that $Y_{\mathrm{pk}} w_{(k)}^{\mu}=0$. Generally, $Y_{i k}$ depends on particle number densities $n_{i}$ and ratios $T / T_{\mathrm{c} i}$, where $T$ is the temperature and $T_{\mathrm{c} i}$ is the critical temperature for transition of particle species $i$ into the superfluid state. The entrainment effect is described by off-diagonal entrainment matrix elements $Y_{\mathrm{np}}=Y_{\mathrm{pn}}$.

Below we write down the linearized equations describing small oscillations, with all perturbations depending on time as $\mathrm{e}^{\imath \sigma t}$ in the frame rotating with the star (with frequency $\Omega$ ). In the Newtonian limit and assuming Cowling approximation these equations consist of (Kantor \& Gusakov 2017):

(i) Continuity equations for baryons and electrons

$\delta n_{\mathrm{b}}+\operatorname{div}\left(n_{\mathrm{b}} \boldsymbol{\xi}_{\mathrm{b}}\right)=0, \quad \delta n_{\mathrm{e}}+\operatorname{div}\left(n_{\mathrm{e}} \boldsymbol{\xi}\right)=0$.

Here and hereafter $\delta$ stands for the Euler perturbation of the corresponding thermodynamic quantity; $\boldsymbol{\xi} \equiv \boldsymbol{j}_{\mathrm{e}} /\left(\imath \sigma n_{\mathrm{e}}\right)$ is the Lagrangian displacement of the normal liquid component (electrons as well as non-superfluid neutrons and protons) ${ }^{1}$, and $\boldsymbol{\xi}_{\mathrm{b}} \equiv \boldsymbol{j}_{\mathrm{b}} /\left(\imath \sigma n_{\mathrm{b}}\right)$ is the Lagrangian displacement for baryons; $n_{\mathrm{b}} \equiv n_{\mathrm{n}}+n_{\mathrm{p}}$ and $\boldsymbol{j}_{\mathrm{b}} \equiv \boldsymbol{j}_{\mathrm{n}}+\boldsymbol{j}_{p}$ are the baryon number density and baryon current density, respectively.

(ii) Euler equation

$-\sigma^{2} \boldsymbol{\xi}_{\mathrm{b}}+2 \imath \sigma \boldsymbol{\Omega} \times \boldsymbol{\xi}_{\mathrm{b}}=\frac{\delta w}{w^{2}} \nabla P-\frac{\boldsymbol{\nabla} \delta P}{w}$,

where $w=(P+\epsilon) / c^{2}, P$ is the pressure, $\epsilon$ is the energy density, and $c$ is the speed of light;

(iii) the 'superfluid' equation, analogue of the Euler equation for superfluid (neutron) liquid component,

$h \sigma^{2} \boldsymbol{z}-2 \imath h_{1} \sigma \boldsymbol{\Omega} \times \boldsymbol{z}=c^{2} n_{\mathrm{e}} \boldsymbol{\nabla} \Delta \mu_{\mathrm{e}}$.

Here $\boldsymbol{z} \equiv \boldsymbol{\xi}_{\mathrm{b}}-\boldsymbol{\xi}$ is the superfluid Lagrangian displacement, $\Delta \mu_{\mathrm{e}} \equiv \mu_{\mathrm{n}}-\mu_{\mathrm{p}}-\mu_{\mathrm{e}}$ is the chemical potential imbalance (note that $\delta \Delta \mu_{\mathrm{e}}=\Delta \mu_{\mathrm{e}}$ since for an unperturbed star $\Delta \mu_{\mathrm{e}}=0$ due to the condition of beta-equilibrium), and quantities $h$ and $h_{1}$ are expressed through the entrainment matrix $Y_{i k}$ as

$h=n_{\mathrm{b}} \mu_{\mathrm{n}} y, \quad h_{1}=\mu_{\mathrm{n}} n_{\mathrm{b}}\left(\frac{n_{\mathrm{b}}}{Y_{\mathrm{nn}} \mu_{\mathrm{n}}+Y_{\mathrm{np}} \mu_{\mathrm{p}}}-1\right), \quad y=\frac{n_{\mathrm{b}} Y_{\mathrm{pp}}}{\mu_{\mathrm{n}}\left(Y_{\mathrm{nn}} Y_{\mathrm{pp}}-Y_{\mathrm{np}}^{2}\right)}-1$.

Note that in the absence of entrainment $\left(Y_{\mathrm{np}}=0\right) h_{1}$ and $h$ are equal, $h_{1}=h$. The 'superfluid' equation takes the form (4) if the interaction between the neutron vortices and normal component is weak (the weak-drag regime), which is true for typical NS conditions (see, e.g., Mendell 1991; Andersson et al. 2006).

The equations (i)-(iii) should be supplemented by the 'equation of state' (EOS), $\delta n_{i}=\frac{\partial n_{i}}{\partial P} \delta P+\frac{\partial n_{i}}{\partial \Delta \mu_{\mathrm{e}}} \Delta \mu_{\mathrm{e}}$.

In the present study we are interested in the inertial oscillation modes, whose eigenfrequencies $\sigma$ vanish as $\Omega \rightarrow 0$. Thus, up to the terms $\sim\left(\Omega / \Omega_{0}\right)^{2}$ ( $\Omega_{0}$ is of the order of Kepler frequency), the eigenfrequency $\sigma$, the Euler perturbation of any (scalar) thermodynamic parameter $f$ (e.g., $P, \mu_{\mathrm{e}}, n_{\mathrm{b}}$ etc.), and the Lagrangian displacement $\boldsymbol{d}$ (e.g., $\boldsymbol{\xi}_{\mathrm{b}}$ or $\boldsymbol{z}$ ) can be presented as (e.g., Provost et al. 1981; Lockitch \& Friedman 1999)

$\sigma=\Omega \sigma_{0}\left(1+\Omega^{2} \sigma_{1}\right)$

$\delta f(t, r, \theta, \phi)=\Omega^{2} \delta f^{1}(r, \theta) \exp (\imath \sigma t+\imath m \phi)$,

$\boldsymbol{d}(t, r, \theta, \phi)=\left[\boldsymbol{d}^{0}(r, \theta)+\Omega^{2} \boldsymbol{d}^{1}(r, \theta)\right] \exp (\imath \sigma t+\imath m \phi)$,

where $m$ is an integer and $(r, \theta, \phi)$ are spherical coordinates with the origin at the stellar center.

In this paper we work in the leading order in $\Omega / \Omega_{0}$, i.e. ignore the terms $\sigma_{1}, \boldsymbol{d}^{1}$ and the stellar oblateness (i.e. all the equilibrium quantities depend on the radial coordinate only). Then the system (i)-(iii) can be represented, in spherical

1 We assume that all non-superfluid components move with one and the same velocity due to efficient particle collisions. 
coordinates, as

$$
\begin{aligned}
& \frac{1}{n_{\mathrm{b}}} \frac{1}{r^{2}} \frac{\partial}{\partial r} r^{2} n_{\mathrm{b}} \xi_{\mathrm{b} r}^{0}+\frac{1}{r \sin \theta}\left(\frac{\partial}{\partial \theta} \sin \theta \xi_{\mathrm{b} \theta}^{0}+\imath m \xi_{\mathrm{b} \phi}^{0}\right)=0, \\
& \frac{1}{n_{\mathrm{e}}} \frac{1}{r^{2}} \frac{\partial}{\partial r} r^{2} n_{\mathrm{e}}\left(\xi_{\mathrm{b} r}^{0}-z_{r}^{0}\right)+\frac{1}{r \sin \theta}\left[\frac{\partial}{\partial \theta} \sin \theta\left(\xi_{\mathrm{b} \theta}^{0}-z_{\theta}^{0}\right)+\imath m\left(\xi_{\mathrm{b} \phi}^{0}-z_{\phi}^{0}\right)\right]=0 \\
& -\sigma_{0}^{2} \xi_{\mathrm{b} r}^{0}-2 \imath \sigma_{0} \sin \theta \xi_{\mathrm{b} \phi}^{0}=-\frac{\partial}{\partial r} \frac{\delta P^{1}}{w}+\frac{\mu_{\mathrm{n}}}{w^{2} c^{2}} \frac{\partial n_{\mathrm{b}}}{\partial \Delta \mu_{\mathrm{e}}} \Delta \mu_{\mathrm{e}}^{1} \frac{d P}{d r}, \\
& -\sigma_{0} \xi_{\mathrm{b} \theta}^{0}-2 \imath \cos \theta \xi_{\mathrm{b} \phi}^{0}=\frac{1}{\imath m} \frac{\partial}{\partial \theta} \sin \theta\left[-\sigma_{0} \xi_{\mathrm{b} \phi}^{0}+2 \imath\left(\xi_{\mathrm{b} r}^{0} \sin \theta+\xi_{\mathrm{b} \theta}^{0} \cos \theta\right)\right], \\
& -\sigma_{0}^{2} \xi_{\mathrm{b} \phi}^{0}+2 \imath \sigma_{0}\left(\xi_{\mathrm{b} r}^{0} \sin \theta+\xi_{\mathrm{b} \theta}^{0} \cos \theta\right)=-\frac{1}{w} \frac{\imath m}{r \sin \theta} \delta P^{1}, \\
& -\sigma_{0}^{2} z_{r}^{0}-2 \imath \frac{h_{1}}{h} \sigma_{0} \sin \theta z_{\phi}^{0}=-\frac{c^{2} n_{\mathrm{e}}}{h} \frac{\partial}{\partial r} \Delta \mu_{\mathrm{e}}^{1}, \\
& -\sigma_{0} z_{\theta}^{0}-2 \imath \frac{h_{1}}{h} \cos \theta z_{\phi}^{0}=\frac{1}{\imath m} \frac{\partial}{\partial \theta} \sin \theta\left[-\sigma_{0} z_{\phi}^{0}+2 \imath \frac{h_{1}}{h}\left(z_{r}^{0} \sin \theta+z_{\theta}^{0} \cos \theta\right)\right] \\
& -\sigma_{0}^{2} z_{\phi}^{0}+2 \imath \frac{h_{1}}{h} \sigma_{0}\left(z_{r}^{0} \sin \theta+z_{\theta}^{0} \cos \theta\right)=-\frac{c^{2} n_{\mathrm{e}}}{h} \frac{\imath m}{r \sin \theta} \Delta \mu_{\mathrm{e}}^{1} .
\end{aligned}
$$

To obtain these equations we (i) substituted (6)-(8) into equations (2)-(4), (ii) omitted higher-order in $\Omega / \Omega_{0}$ terms, (iii) substituted $\delta P^{1}$ from the $\phi$-component of the Euler equation (13) into the $\theta$-component of the Euler equation, (iv) substituted $\Delta \mu_{\mathrm{e}}^{1}$ from the $\phi$-component of superfluid equation (16) into the $\theta$-component of the superfluid equation, (v) expressed $\delta w$ in (11) through $\delta P$ and $\Delta \mu_{\mathrm{e}}$ (see Kantor \& Gusakov 2017, Appendix A), and (vi) divided superfluid equation by $-h(r)$.

It is convenient to express the functions $\xi_{\mathrm{b} \theta}^{0}, \xi_{\mathrm{b} \phi}^{0}, z_{\theta}^{0}$, and $z_{\phi}^{0}$ in the system (9)-(16) as a sum of toroidal $\left(T, T_{z}\right)$ and poloidal $\left(Q, Q_{z}\right)$ components (Saio 1982):

$\xi_{\mathrm{b} \theta}^{0}=\frac{\partial}{\partial \theta} Q(r, \theta)+\frac{\imath m T(r, \theta)}{\sin \theta}, \quad \xi_{\mathrm{b} \phi}^{0}=\frac{\imath m Q(r, \theta)}{\sin \theta}-\frac{\partial}{\partial \theta} T(r, \theta)$,

$z_{\theta}^{0}=\frac{\partial}{\partial \theta} Q_{z}(r, \theta)+\frac{\imath m T_{z}(r, \theta)}{\sin \theta}, \quad z_{\phi}^{0}=\frac{\imath m Q_{z}(r, \theta)}{\sin \theta}-\frac{\partial}{\partial \theta} T_{z}(r, \theta)$.

Then, following the same procedure as for non-superfluid stars (e.g., Lockitch \& Friedman 1999), we expand all the unknown functions into Legendre polynomials with fixed $m$ :

$$
\begin{aligned}
& \xi_{\mathrm{b} r}^{0}(r, \theta)=\sum_{l_{2}} \xi_{\mathrm{b} r l_{2} m}^{0}(r) P_{l_{2}}^{m}(\cos \theta), \\
& z_{r}(r, \theta)=\sum_{l_{2}} z_{r l_{2} m}^{0}(r) P_{l_{2}}^{m}(\cos \theta), \\
& Q(r, \theta)=\sum_{l_{2}} Q_{l_{2} m}(r) P_{l_{2}}^{m}(\cos \theta), \\
& Q_{z}(r, \theta)=\sum_{l_{2}} Q_{z l_{2} m}(r) P_{l_{2}}^{m}(\cos \theta), \\
& T(r, \theta)=\sum_{l_{1}} T_{l_{1} m}(r) P_{l_{1}}^{m}(\cos \theta), \\
& T_{z}(r, \theta)=\sum_{l_{1}} T_{z} l_{1} m(r) P_{l_{1}}^{m}(\cos \theta), \\
& \delta P^{1}(r, \theta)=\sum_{l_{2}} \delta P_{l_{2} m}^{1}(r) P_{l_{2}}^{m}(\cos \theta), \\
& \Delta \mu_{\mathrm{e}}^{1}(r, \theta)=\sum_{l_{2}} \Delta \mu_{\mathrm{e} l_{2} m}^{1}(r) P_{l_{2}}^{m}(\cos \theta),
\end{aligned}
$$

where the summation goes over $l_{1}=m+2 k$ and $l_{2}=m+2 k+1(k=0,1,2, \ldots)$ for 'odd' modes, and over $l_{1}=m+2 k+1$, $l_{2}=m+2 k$ for 'even' modes. ${ }^{2}$ After substituting these expansions into oscillation equations, one obtains an infinite set of ordinary differential equations for unknown functions $\xi_{\mathrm{b} r l_{2} m}^{0}(r), z_{r l_{2} m}^{0}(r), \ldots$

The oscillation equations should be supplemented by the boundary conditions, which consist of:

(i) regularity condition for the perturbations in the stellar center,

2 Following Yoshida \& Lee (2000), we call 'even' the modes whose scalar perturbations are symmetric with respect to the equator, and 'odd' - the modes with asymmetric perturbations. Odd and even modes are completely decoupled and do not mix with each other. 

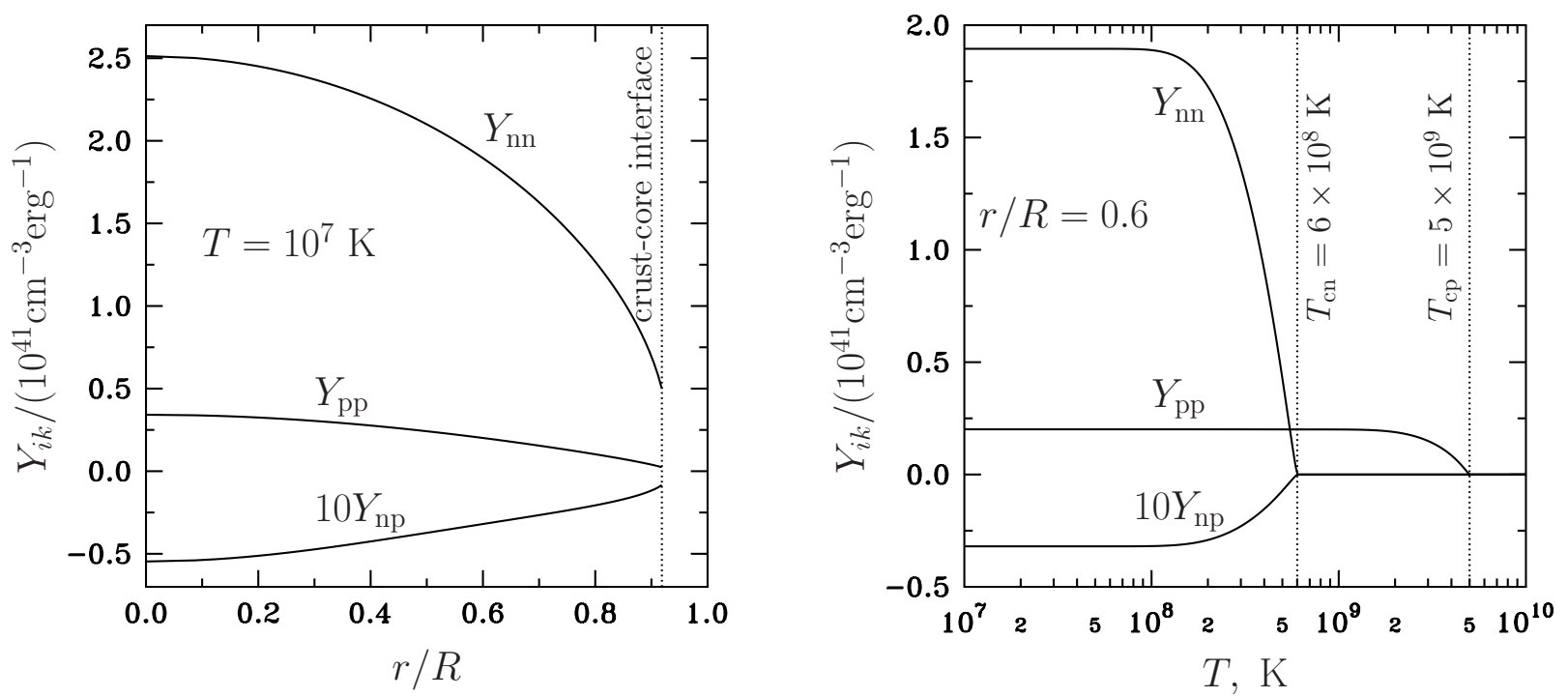

Figure 1. Elements of the entrainment matrix $Y_{i k}$ (in units of $10^{41} \mathrm{~cm}^{-3} \mathrm{erg}^{-1}$ ) versus $r / R$ at $T=10^{7} \mathrm{~K}$ (left panel) and versus temperature $T$ at $r / R=0.6$ (right panel). Critical temperatures are constant throughout the core, $T_{\mathrm{cn}}=6 \times 10^{8} \mathrm{~K}, T_{\mathrm{cp}}=5 \times 10^{9} \mathrm{~K}$.

(ii) vanishing of the Lagrangian perturbation of the pressure at the stellar surface $r=R$,

$\delta P(R)+\left.\xi_{\mathrm{b} r}(R) \frac{d P}{d r}\right|_{r=R}=0$

(iii) continuity of $\delta P, \xi_{\mathrm{b} r}$ and $\xi_{r}$ at the superfluid/non-superfluid interface.

\section{CLASSIFICATION OF ROTATIONAL MODES}

We consider rotational oscillation modes with $\sigma \propto \Omega$ in the slow-rotation approximation (Lockitch \& Friedman 1999; Yoshida \& Lee 2000 ). Each mode is characterized by two angular 'quantum numbers', $l_{0}$ and $m$, where $m$ is azimuthal number and $l_{0}$ (in the notation by Lindblom \& Ipser 1999; Yoshida \& Lee 2000) is the maximum index $l$ of spherical harmonics associated with the dominant expansion coefficients of the eigenfunctions. For the uniform density stars all coefficients with $l>l_{0}$ are strictly zero (Lockitch \& Friedman 1999).

For a given $m$, there are two nodeless modes with $l_{0}-|m|=1$ : the purely toroidal normal r-mode with $\sigma_{0}=2 /(m+1)$, and the superfluid r-mode, which, in the limit $Y_{\mathrm{np}}=0$, is also purely toroidal and has the same frequency (Andersson \& Comer 2001; Lee \& Yoshida 2003; Andersson et al. 2009; Kantor \& Gusakov 2017). For a given $m$ and $l_{0}>|m|+1$ there are $l_{0}-|m|$ normal inertial modes $\left(i^{\circ}\right.$-modes) and $l_{0}-|m|$ superfluid inertial modes $\left(i^{s}\right.$-modes). The modes where normal and superfluid components are comoving, so that $\left|\boldsymbol{\xi}_{\mathrm{b}}\right| \sim|\boldsymbol{z}|$, are called 'normal', or 'ordinary', and denoted with a superscript ${ }^{\circ}$; If normal and superfluid components are counter-moving, so that the total baryon current is almost not excited, $\left|\boldsymbol{\xi}_{\mathrm{b}}\right| \ll|\boldsymbol{z}|$, then the corresponding modes are referred to as 'superfluid', and designated with a superscript ${ }^{s}$. The number of radial nodes in eigenfunctions of a given mode is determined by $l_{0}$ and $m$ (see Yoshida \& Lee 2000, Table 3). For example, the dominant lowest-order toroidal eigenfunction $\left[T_{m m}(r)\right.$ for $i^{o}$-modes, and $T_{z m m}(r)$ for $i^{s}$-modes] has no nodes for the $r$-mode $\left(l_{0}-|m|=1\right)$, one node for $l_{0}-|m|=3$ mode, and two nodes for $l_{0}-|m|=5$ mode.

\section{RESULTS FOR THE SPECTRUM}

In our numerical calculations we adopt the parametrization of Heiselberg \& Hjorth-Jensen (1999) of APR equation of state (Akmal et al. 1998) for the NS core, and the equation of state BSk20 (Potekhin et al. 2013) for the crust. All calculations are performed for an NS with mass $M=1.4 M_{\odot}$ and radius $R=12.18 \mathrm{~km}$. To calculate the unperturbed model of a star, we use the Tolman-Oppenheimer-Volkoff equations. ${ }^{3}$ We also assume that the baryon critical temperatures are constant throughout

3 Note that here we, as Kantor \& Gusakov (2017), use relativistic background NS model and relativistic EOS, but Newtonian oscillation equations. One should bear in mind that this inconsistency may, in principle, affect the results of our calculations. 


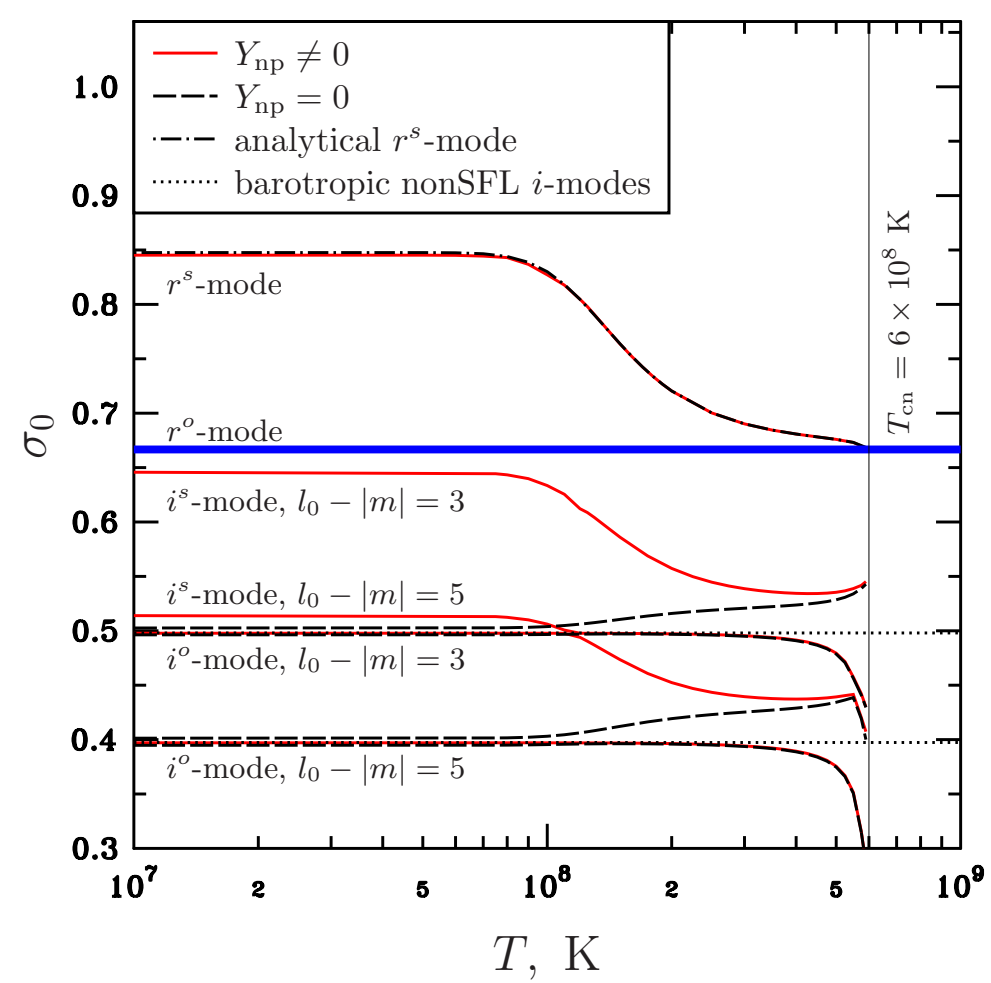

Figure 2. Eigenfrequency $\sigma_{0}$ versus stellar temperature $T$ for $m=2$ inertial modes. Critical temperatures are constant throughout the core, $T_{\mathrm{cn}}=6 \times 10^{8} \mathrm{~K}, T_{\mathrm{cp}}=5 \times 10^{9} \mathrm{~K}$. Solid lines denote inertial modes calculated taking into account the entrainment effect $\left(Y_{\mathrm{np}} \neq 0\right)$, dashed lines denote the same modes calculated without the entrainment effect. Dot-dashed line denotes the superfluid $r^{s}$-mode calculated via the approximate analytical method introduced in Section 5. Dotted lines correspond to $l_{0}-|m|=3$ and $l_{0}-|m|=5$ normal $i$-modes in a non-superlfuid barotropic NS with the same EOS and mass. In all cases the $m=2$ normal $r$-mode (bold line) has the same frequency $\sigma_{0}=2 / 3$.

the core: $T_{\mathrm{cn}}=6 \times 10^{8} \mathrm{~K}, T_{\mathrm{cp}}=5 \times 10^{9} \mathrm{~K}$ in all figures except for Fig. 4 , where we set $T_{\mathrm{cn}}=5 \times 10^{9} \mathrm{~K}, T_{\mathrm{cp}}=5 \times 10^{8} \mathrm{~K}$. The entrainment matrix $Y_{\mathrm{ik}}$ is calculated in a way similar to how it was done in Kantor \& Gusakov (2011). The elements of this matrix are shown in Fig. 1 as functions of radial coordinate $r / R$ at temperature $T=10^{7} \mathrm{~K}$ (left panel) and as functions of temperature $T$ at fixed $r / R=0.6$ (right panel).

The key ingredients of the scenario proposed by Gusakov et al. (2014a,b) are the avoided crossings of inertial modes with the $m=2$ normal $r$-mode. We calculated only the modes that could interact with this mode i.e. the odd $\left(l_{0}-|m|=1\right.$, $l_{0}-|m|=3$, and $\left.l_{0}-|m|=5\right) m=2$ inertial modes. ${ }^{4}$ We do not look for the modes with eigenfrequencies that are too far from the $r$-mode frequency $\left(\sigma_{0}=2 /(m+1)=2 / 3\right.$ in the frame rotating with the star). In order to solve the oscillation equations (9)-(16) numerically, we disregard all the terms with $l>|m|+2 k_{\max }-1$ in the Legendre polynomial expansion. We set $k_{\max }=3$ to calculate $l_{0}-|m|=3$ and $l_{0}-|m|=5$ modes. This value allows us to reproduce the results of Yoshida \& Lee (2000) for the $l_{0}-|m|=3$ inertial modes within the accuracy of $0.2 \%$. For r-modes $\left(l_{0}-|m|=1\right)$, for which only $l=m$ and $l=m+1$ harmonics are significant, we set $k_{\max }=2$.

In Fig. 2 we show the spectrum for $l_{0}-|m|=1, l_{0}-|m|=3$, and $l_{0}-|m|=5$ inertial modes. Dashed lines denote the i-modes calculated without entrainment $\left(Y_{\mathrm{np}}=0\right)$, solid lines - with entrainment. The bold line is the normal $m=2 r^{\circ}$-mode, $\sigma_{0}=2 / 3$ (note that if $Y_{\mathrm{np}}=0$ the superfluid r-mode has the same frequency in the lowest order in $\Omega$, see Andersson \& Comer 2001; Lee \& Yoshida 2003; Andersson et al. 2009; Kantor \& Gusakov 2017). The dot-dashed line denotes the superfluid rmode calculated analytically via the approximate method described in Section 5 . This approximate method accounts for the first-order terms in small parameter $\Delta h \equiv h_{1} / h-1$ and thus it is accurate up to the terms $\sim \Delta h^{2}$. In Fig. 3 we plot the ratio $h_{1}(r) / h(r)$ for our NS model at $T=10^{7} \mathrm{~K}$ (dashed line) and $T=5.5 \times 10^{8} \mathrm{~K}$ (dot-dashed line). As one can see from this Figure, $\Delta h$ at low temperatures is much larger than at temperatures close to $T_{\text {cn }}: \Delta h \gtrsim 0.2$ at $T=10^{7} \mathrm{~K}, \Delta h \lesssim 0.02$ at

4 The odd modes with higher $l_{0}$, which also can interact with the $r^{\circ}$-mode, are harder to calculate numerically, so in the present study we focus only on the modes with low $l_{0}$. 


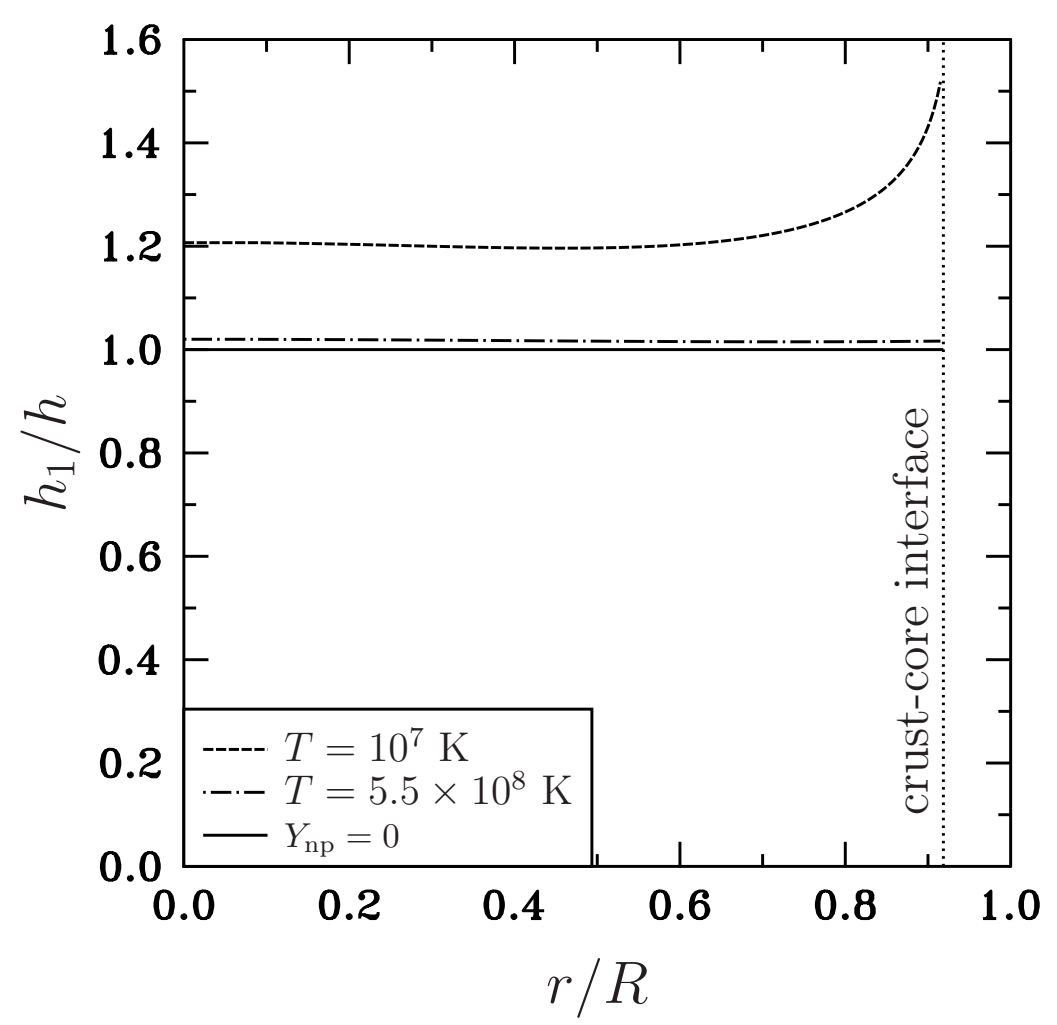

Figure 3. The ratio $h_{1} / h$ versus normalized radial coordinate $r / R$ for temperatures $T=10^{7} \mathrm{~K}$ (dashed line) and $T=5.5 \times 10^{8} \mathrm{~K}($ dotdashed line). Critical temperatures are constant throughout the core, $T_{\mathrm{cn}}=6 \times 10^{8} \mathrm{~K}, T_{\mathrm{cp}}=5 \times 10^{9} \mathrm{~K}$. In the absence of entrainment $\left(Y_{\mathrm{np}}=0\right) h_{1} / h \equiv 1$ (solid line).

$T=5.5 \times 10^{8} \mathrm{~K}$. Therefore one can expect that at $T \rightarrow T_{\mathrm{cn}}$ the $r^{s}$-mode frequency will approach the value $\sigma_{0}=2 /(m+1)$, and the accuracy of our analytical method will increase. Indeed, this conclusion is confirmed in Fig. 2. One can see that even at low temperatures, when the frequency of $r^{s}$-mode significantly differs from the normal $r^{o}$-mode frequency, the two methods of calculating the frequency of $r^{s}$-mode give the same result within the accuracy of $1 \%$. For example, at $T=10^{7} \mathrm{~K}$ numerical calculation for $r^{s}$-mode yields $\sigma_{0}=0.8452$, while the analytical result is $\sigma_{0}=0.8476$. At higher temperatures $T>3 \times 10^{8} \mathrm{~K}$ the relative difference between numerical and analytical results does not exceed $0.01 \%$. Note, however, that the approximate method does not provide such a good accuracy for eigenfunctions (see discussion of Fig. 7 below).

The spectrum of inertial modes exhibits some interesting features: (i) At low temperatures in the absence of entrainment the frequencies of the normal and superfluid i-modes almost coincide. (ii) While the entrainment effect almost does not affect the normal inertial modes $\left(i^{o}\right.$-modes), it noticeably pushes the frequencies of superfluid modes up. We discuss this behavior in Section 6. In order to illustrate the transition from the case $Y_{\mathrm{np}} \neq 0$ to $Y_{\mathrm{np}}=0$, we show in Fig. 4 the spectrum for the same modes, but employing another (non-realistic) ordering of critical temperatures: $T_{\mathrm{cp}}=5 \times 10^{8} \mathrm{~K}, T_{\mathrm{cn}}=5 \times 10^{9} \mathrm{~K}$. The absolute value of $Y_{\mathrm{np}}$ decreases as the temperature goes up, and vanishes at $T=T_{\mathrm{cp}}$, when all protons become non-superconducting. (iii) In contrast to the normal $r^{\circ}$-mode, which has the same frequency in the whole temperature range in both superfluid and non-superfluid NSs, the frequencies of the normal $i^{\circ}$-modes at temperatures close to $T_{\mathrm{cn}}$ do not remain constant but go down. This is not surprising, since non-superfluid stratified npe-matter of NS cores does not support inertial modes. Superfluid $i^{s}$-mode frequencies, in contrast, grow at $T \rightarrow T_{\mathrm{cn}}$. In order to explain all these features, in Section 6 we analyze a dispersion relation for inertial modes in short-wavelength limit (see equations 73-77 and their discussion).

In Fig. 2 one can see avoided crossings of the $l_{0}-|m|=3$ normal $i^{o}$-mode and its neighbouring $l_{0}-|m|=5$ superfluid $i^{s}$-mode (see solid lines) at $T \sim 10^{8} \mathrm{~K}$ and at $T \sim 5.5 \times 10^{8} \mathrm{~K}$ : normal mode transforms into superfluid, and vice versa. We did not find any avoided crossing between superfluid $i^{s}$-modes and the normal $r^{o}$-mode, i.e. did not find the stability peaks for the scenario proposed by Gusakov et al. (2014a,b). However, there should be an interaction between the normal and superfluid r-modes at $T \rightarrow T_{\mathrm{cn}}$, which can stabilize normal $r^{\circ}$-mode, and result in the formation of the stability peak at $T=T_{\mathrm{cn}}$. Notice also that at low temperatures the frequency of $l_{0}-|m|=3$ superfluid $i^{s}$-mode is rather close to the frequency of the normal $r^{o}$-mode, which may also lead to stabilizing interaction of modes at low temperatures. To work out these interactions, one has to go beyond the leading-order calculations in $\Omega$, which is out of the scope of the present paper. One may expect to find 


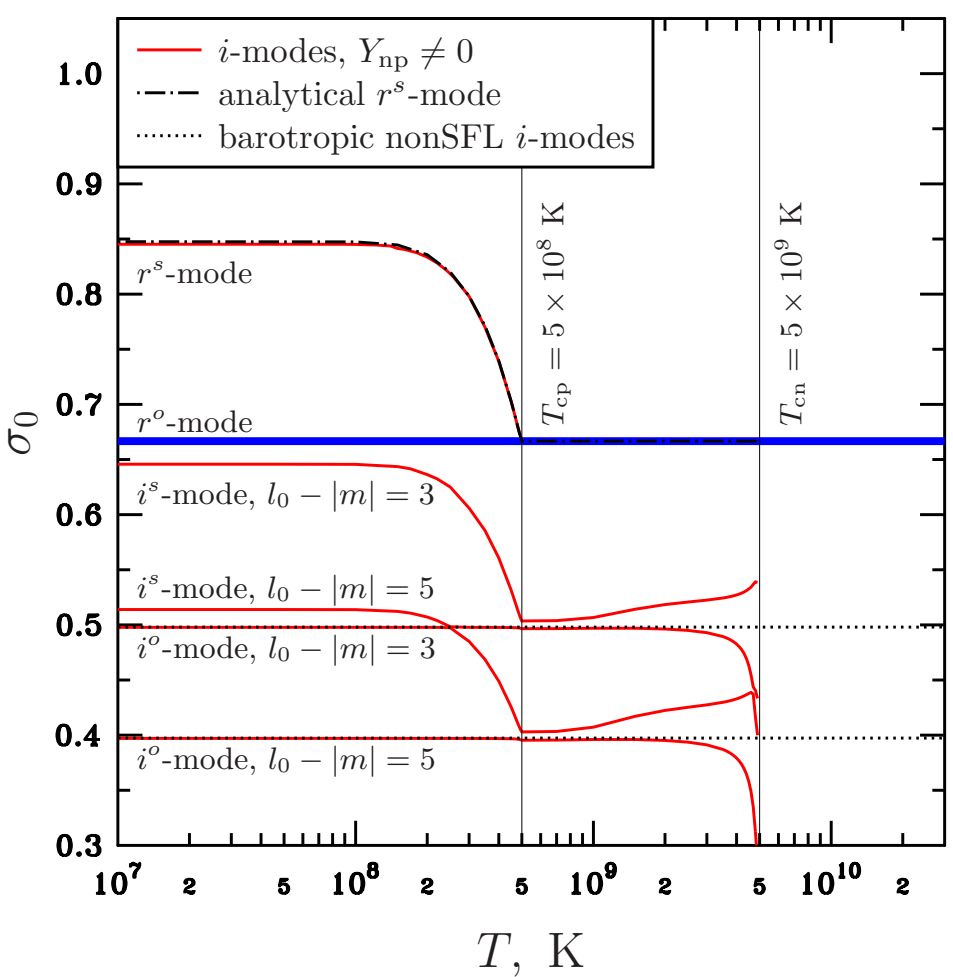

Figure 4. Eigenfrequency $\sigma_{0}$ versus stellar temperature $T$ for $m=2$ inertial modes. Critical temperatures are constant throughout the core, $T_{\mathrm{cn}}=5 \times 10^{9} \mathrm{~K}, T_{\mathrm{cp}}=5 \times 10^{8} \mathrm{~K}$. Solid lines denote inertial modes calculated taking into account the entrainment effect $\left(Y_{\mathrm{np}} \neq 0\right.$ at $T<T_{\mathrm{cp}}$ ), Dot-dashed line denotes the superfluid $r^{s}$-mode calculated via the approximate analytical method introduced in Section 5 . Dotted lines correspond to $l_{0}-|m|=3$ and $l_{0}-|m|=5$ normal $i$-modes in a non-superlfuid barotropic NS with the same EOS and mass. In all cases the $m=2$ normal $r$-mode (bold line) has the same frequency $\sigma_{0}=2 / 3$.
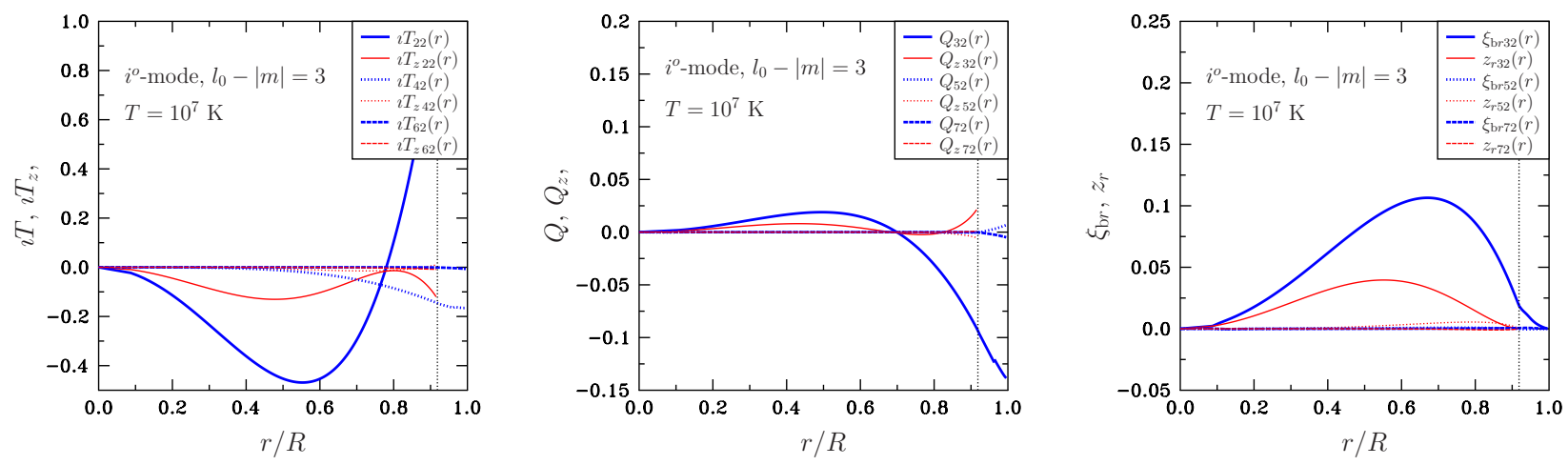

Figure 5. The lowest three harmonics for toroidal (left panel), poloidal (central panel) and radial (right panel) displacements for the $m=2, l_{0}-|m|=3$ normal $i^{\circ}$-mode at $T=10^{7} \mathrm{~K}$. Bold lines denote Lagrangian displacements for baryons, and thin lines denote superfluid displacements. Critical temperatures are constant throughout the core, $T_{\mathrm{cn}}=6 \times 10^{8} \mathrm{~K}, T_{\mathrm{cp}}=5 \times 10^{9} \mathrm{~K}$. Vertical dots show the crust-core interface.

avoided crossings of $r^{o}$-mode with another inertial modes under more realistic assumptions about the NS composition, and/or for modes with larger $l_{0}$. In the future work we are going to calculate the spectrum using realistic critical temperature profiles and accounting for muons, which play very important role in defining oscillation spectrum (Kantor \& Gusakov 2017).

In order to illustrate the properties of inertial modes, we also plot their eigenfunctions. Fig. 5 shows eigenfunctions for $2 \leq l \leq 7$ harmonics of toroidal, poloidal and radial displacements for $l_{0}-|m|=3 i^{\circ}$-mode at $T=10^{7} \mathrm{~K}$. One can see that the baryon displacements (bold lines) are larger than the superfluid ones (thin lines). We also see that each of the dominant 

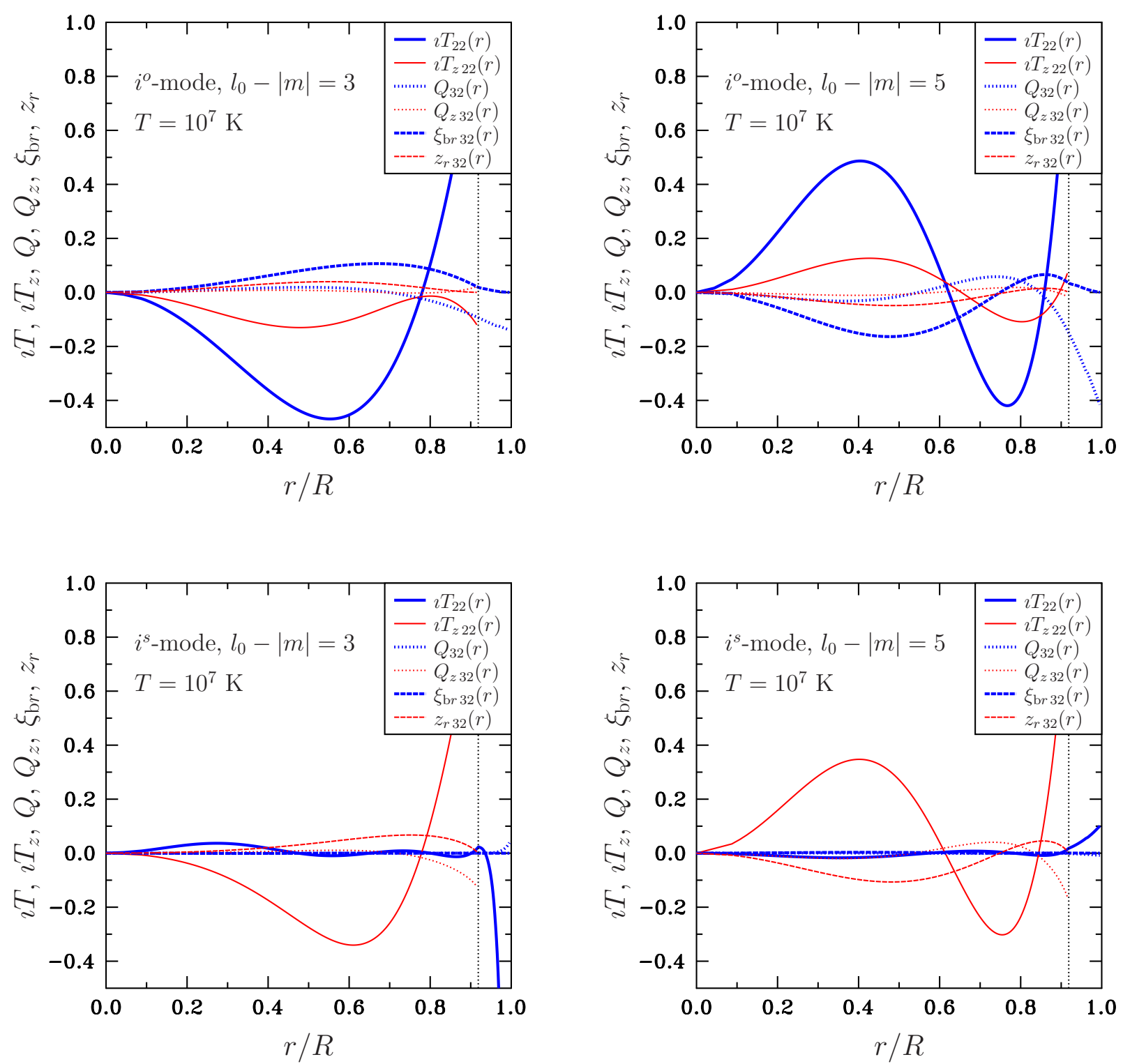

Figure 6. Eigenfunctions for different $m=2$ inertial modes at $T=10^{7} \mathrm{~K}$. Top left: $l_{0}-|m|=3$ normal $i^{o}$-mode. Top right: $l_{0}-|m|=5$ normal $i^{o}$-mode. Bottom left: the $l_{0}-|m|=3$ superfluid $i^{s}$-mode. Bottom right: the $l_{0}-|m|=5$ superfluid $i^{s}$-mode. Only lowest-order harmonics $\left(l=2\right.$ for toroidal displacements $T, T_{z}$ and $l=3$ for poloidal and radial displacements $\left.Q, Q_{z}, \xi_{\mathrm{b} r}, z_{r}\right)$ are plotted. Bold lines denote Lagrangian displacements for baryons, and thin lines denote superfluid displacements. Critical temperatures are constant throughout the core, $T_{\mathrm{cn}}=6 \times 10^{8} \mathrm{~K}, T_{\mathrm{cp}}=5 \times 10^{9} \mathrm{~K}$. Vertical dots show the crust-core interface.

eigenfunctions $T_{22}(r), Q_{32}(r)$ and $\xi_{\mathrm{b} r 32}(r)$ have one radial node, ${ }^{5}$ in accordance with Table 3 in the paper by Yoshida \& Lee (2000).

The lowest harmonics for $l_{0}-|m|=3$ and $l_{0}-|m|=5$ normal and superfluid $i$-modes are plotted in Fig. 6 . Eigenfunctions are normalized so that $\imath T_{22}\left(R_{c c}\right)=1$ for $i^{o}$-modes, and $\imath T_{z 22}\left(R_{c c}\right)=1$ for $i^{s}$-modes. One can clearly see the key difference between the normal and superfluid modes: for normal modes (top left and top right panel) baryon displacements (bold lines) are comparable with (and even larger than) the superfluid ones (thin lines), while for superfluid modes (bottom left and bottom right panel) superfluid displacements dominate. It is also interesting that toroidal displacements are larger than poloidal for all considered modes. The lowest-order dominant eigenfunctions $\left(T_{22}, Q_{32}\right.$ and $\xi_{\mathrm{b} r 32}$ for $i^{o}$-modes, $T_{z 22}, Q_{z 32}$ and $z_{r 32}$ for $i^{s}$-modes) have one radial node for the case $l_{0}-|m|=3$ (left) and two nodes for $l_{0}-|m|=5$ - again, as expected from Table 3

5 Following Yoshida \& Lee (2000), we include the node at the stellar surface in the count of nodes. 

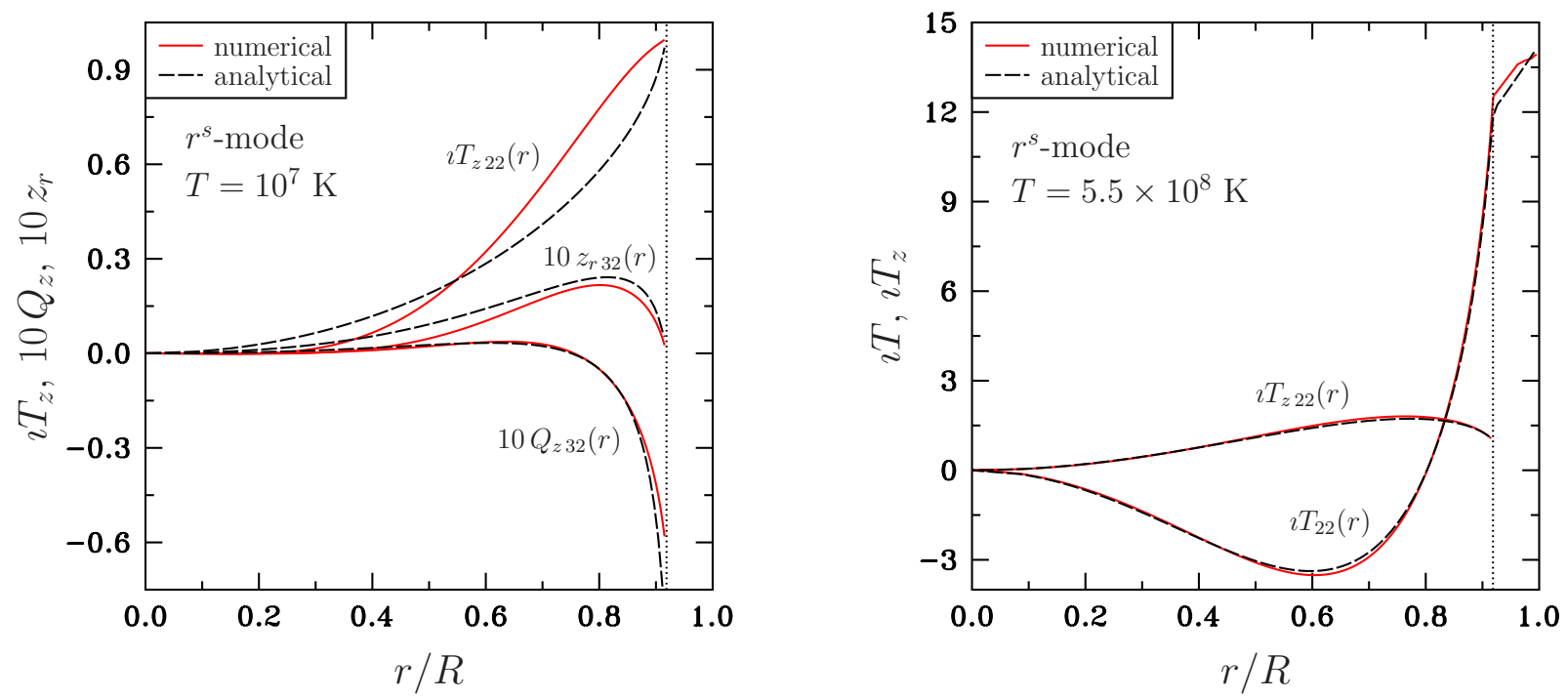

Figure 7. Dominant eigenfunctions for the $m=2$ superfluid $r$-mode, obtained by numerical calculations (solid lines) and by the approximate analytical method (dashed lines). Left panel displays superfluid displacements $T_{z 22}, Q_{z} 32$ (multiplied by 10), and $z_{r} 32$ (multiplied by 10) for $T=10^{7} \mathrm{~K}$. Right panel displays toroidal displacements $T_{z 22}$ and $T_{22}$ for $T=5.5 \times 10^{8} \mathrm{~K}$. Critical temperatures are constant throughout the core, $T_{\mathrm{cn}}=6 \times 10^{8} \mathrm{~K}, T_{\mathrm{cp}}=5 \times 10^{9} \mathrm{~K}$. Vertical dots show the crust-core interface.

in the paper by Yoshida \& Lee (2000). Note that, e.g., $T_{22}(r)$ for the $l_{0}-|m|=3 i^{s}$-mode has more nodes than $T_{z 22}(r)$ and cannot be used to determine the value of $l_{0}$.

We also compare eigenfunctions obtained via numerical calculations and via analytical method from Section 5 for the superfluid $r^{s}$-mode at low $\left(T=10^{7} \mathrm{~K}\right)$ and high $\left(T=5.5 \times 10^{8} \mathrm{~K}\right)$ temperatures. The results are shown in Fig. 7 . Solid lines represent the numerical results, dashed lines show analytical results. For $T=10^{7} \mathrm{~K}$ (left panel) we plotted only the superfluid displacements $T_{z 22}(r), Q_{z 32}$ and $z_{r 32}$ because they are much larger than the baryon ones. Since the toroidal component is dominating, we multiplied $Q_{z 32}$ and $z_{r 32}$ by 10 in order to make them visible. We see that the numerical and analytical methods lead to qualitatively similar, but quantitatively different result: while the eigenfrequencies coincide within the accuracy of $1 \%$, eigenfunctions differ significantly. At $T=5.5 \times 10^{8} \mathrm{~K}$ (right panel), where entrainment effect is small (see Fig. 3) and the $r^{s}$-mode frequency is close to that of $r^{o}$-mode, analytical method becomes more accurate. Indeed, we see that in this case the eigenfunctions coincide much better, within the accuracy of $10 \%$. Here we plotted the superfluid and baryon toroidal displacements, $T_{z 22}(r)$ and $T_{22}(r)$, since at this temperature they are much larger than all other eigenfunctions. The large value of $T_{22}(r)$ indicates a possible interaction with the normal $r$-mode. From the analysis of Figs. 2, 4 and 7 we conclude that the approximate method of calculating the superfluid $r$-mode gives a very good accuracy (better than $1 \%$ ) for eigenfrequencies, but one has to keep in mind that the eigenfunctions are calculated much less accurately, especially when entrainment effect is large (e.g., at low temperatures).

\section{SUPERFLUID R-MODE IN THE LIMIT OF SMALL ENTRAINMENT}

In this section we provide an approximate method that allows one to calculate the superfluid $r$-mode in npe NS analytically in the lowest order in $\Omega$, accounting for entrainment effect. If there is no entrainment $\left(Y_{\mathrm{np}}=0\right.$ and thus $h_{1}=h$, see equation 5 ), then for a given $m$ there exist two purely toroidal rotational modes, the normal $r$-mode and the superfluid $r$-mode, both having (to the lowest order in $\left.\Omega / \Omega_{0}\right)$ the same frequency $\sigma_{0}=2 /(m+1)$ (Andersson \& Comer 2001; Lee \& Yoshida 2003; Andersson et al. 2009; Kantor \& Gusakov 2017). If the entrainment effect is present, then, except for some degenerate cases when $h_{1}$ is equal to $h,{ }^{6}$ the superfluid $r$-mode turns into a mixed poloidal-toroidal mode with different frequency. ${ }^{7}$ Assuming that the entrainment effect is small, one can develop a perturbation theory in $\Delta h \equiv h_{1} / h-1$, and analytically find corrections to the eigenfrequency and eigenfunctions for the superfluid $r$-mode in the first order in $\Delta h$. This method is analogous to that of Kantor \& Gusakov (2017), who showed that in npe matter, $r$-modes can be calculated analytically in the next-to-leading

6 As follows from equation 5, the condition $h_{1}=h$ is equivalent to $\mu_{\mathrm{p}} Y_{\mathrm{np}}=-\mu_{\mathrm{n}} Y_{\mathrm{np}}^{2} / Y_{\mathrm{pp}}$. This condition can be, in principle, satisfied even if $Y_{\mathrm{np}} \neq 0$.

7 If $h_{1}(r) / h(r)$ is constant throughout the core, the mode has different frequency but remains purely toroidal. 
order in $\Omega$, but ignoring the entrainment effect (and thus assuming $h_{1}=h$ ). Provost et al. (1981) used a similar approach for analysis of $r$-modes in non-superfluid stars.

Let us start with purely toroidal oscillations, described by system (9)-(16). In that case radial displacements vanish $\left(\xi_{\mathrm{b} r}=z_{r}=0\right)$, and the continuity equations (9) and (10) reduce to

$\frac{\partial}{\partial \theta} \sin \theta \xi_{\mathrm{b} \theta}^{0}+\imath m \xi_{\mathrm{b} \phi}^{0}=0$,

$\frac{\partial}{\partial \theta} \sin \theta z_{\theta}^{0}+\imath m z_{\phi}^{0}=0$.

$\theta$-components of Euler equation (12) and superfluid equation (15) read

$-\sigma_{0} \xi_{\mathrm{b} \theta}^{0}-2 \imath \cos \theta \xi_{\mathrm{b} \phi}^{0}=\frac{1}{\imath m} \frac{\partial}{\partial \theta} \sin \theta\left(-\sigma_{0} \xi_{\mathrm{b} \phi}^{0}+2 \imath \xi_{\mathrm{b} \theta}^{0} \cos \theta\right)$,

$-\sigma_{0} z_{\theta}^{0}-2 \imath \frac{h_{1}}{h} \cos \theta z_{\phi}^{0}=\frac{1}{\imath m} \frac{\partial}{\partial \theta} \sin \theta\left(-\sigma_{0} z_{\phi}^{0}+2 \imath \frac{h_{1}}{h} z_{\theta}^{0} \cos \theta\right)$.

The solution to the system of equations (28) and (30) is

$\sigma_{0}=\frac{2 m}{l(l+1)}, \quad \xi_{\mathrm{b} \theta}^{0}=\frac{\imath m}{\sin \theta} T_{l m}(r) P_{l}^{m}(\cos \theta), \quad \xi_{\mathrm{b} \phi}^{0}=-T_{l m}(r) \frac{d}{d \theta} P_{l}^{m}(\cos \theta)$,

which is the well-known normal $r$-mode. Taking into account the $r$ - and $\phi$-components of Euler equation (equations 11 and $13)$, one can check that only the solution with $l=m$ exists.

The second pair of equations, (29) and (31), describes the superfluid $r$-mode,

$\sigma_{0}=\frac{2 m}{l(l+1)} \frac{h_{1}(r)}{h(r)}, \quad z_{\theta}^{0}=\frac{\imath m}{\sin \theta} T_{z l m}(r) P_{l}^{m}(\cos \theta), \quad z_{\phi}^{0}=-T_{z l m}(r) \frac{d}{d \theta} P_{l}^{m}(\cos \theta)$.

If $Y_{\mathrm{np}}=0$, then $h_{1}=h$, and $\sigma_{0}=\frac{2 m}{l(l+1)}$ is the global oscillation frequency and the superfluid $r$-mode is indeed purely toroidal (and, as for the normal $r$-mode, only $l=m$ solution exists). However, if the entrainment effect is present, $h_{1}(r) / h(r)$ in general case varies throughout the star. This means that the purely toroidal superfluid mode cannot exist, and an admixture of poloidal component is required.

Now let us write down a perturbation theory in $\Delta h \equiv h_{1} / h-1$. Below we denote the zeroth-order in $\Delta h$ quantities with index (0), and the first-order in $\Delta h$ quantities - with index (1).

In this notation, the eigenfrequency $\sigma_{0}$ and the eigenfunctions can be expanded in Taylor series in $\Delta h$ :

$\sigma_{0}=\sigma_{0(0)}+\sigma_{0(1)}+O\left(\Delta h^{2}\right)=\frac{2}{m+1}+\sigma_{0(1)}+O\left(\Delta h^{2}\right)$,

$\xi_{\mathrm{b} r}^{0}=\xi_{\mathrm{b} r}^{0(1)}+O\left(\Delta h^{2}\right), \quad T=T^{(0)}+T^{(1)}+O\left(\Delta h^{2}\right), \quad Q=Q^{(1)}+O\left(\Delta h^{2}\right)$,

$z_{r}^{0}=z_{r}^{0(1)}+O\left(\Delta h^{2}\right), \quad T_{z}=T_{z}^{(0)}+T_{z}^{(1)}+O\left(\Delta h^{2}\right), \quad Q_{z}=Q_{z}^{(1)}+O\left(\Delta h^{2}\right)$,

$\delta P=\delta P^{1(0)}+\delta P^{1(1)}+O\left(\Delta h^{2}\right)$,

$\Delta \mu_{\mathrm{e}}^{1}=\Delta \mu_{\mathrm{e}}^{1(0)}+\Delta \mu_{\mathrm{e}}^{1(1)}+O\left(\Delta h^{2}\right)$.

Since in absence of entrainment the superfluid $r$-mode is purely toroidal, the radial and poloidal displacements in the zeroth order vanish, $\xi_{\mathrm{b} r}^{0(0)}=z_{r}^{0(0)}=Q^{(0)}=Q_{z}^{(0)}=0$.

\subsection{Zero-order solution}

In the zeroth order in $\Delta h$ (i.e. without the entrainment effect) one has to find the eigenfrequency $\sigma_{0(0)}$ and four eigenfunctions $T^{(0)}, T_{z}^{(0)}, \delta P^{1(0)}, \Delta \mu_{\mathrm{e}}^{1(0)}$. As discussed above (see equations 32 and 33), the frequency equals to

$\sigma_{0(0)}=\frac{2}{m+1}$

and the toroidal displacements are proportional to the $l=m$ Legendre polynomial,

$T^{(0)}=T_{m m}^{(0)}(r) P_{m}^{m}(\cos \theta), \quad T_{z}^{(0)}=T_{z m m}^{(0)}(r) P_{m}^{m}(\cos \theta)$.

One can find from equations (11), (13), (14), and (16) that the perturbations $\delta P^{1(0)}$ and $\Delta \mu_{\mathrm{e}}^{1(0)}$ are proportional to the $l=m+1$ Legendre polynomial,

$\delta P^{1(0)}=\delta P_{m+1, m}^{1(0)}(r) P_{m+1}^{m}(\cos \theta), \quad \Delta \mu_{\mathrm{e}}^{1(0)}=\Delta \mu_{\mathrm{e} m+1, m}^{1(0)}(r) P_{m+1}^{m}(\cos \theta)$,

while the coefficients $\delta P_{m+1, m}^{1(0)}(r), \Delta \mu_{\mathrm{e} m+1, m}^{1(0)}(r)$ are expressed through $T_{m m}^{(0)}(r)$ and $T_{z m m}^{(0)}(r)$, respectively:

$\delta P_{m+1, m}^{1(0)}(r)=\frac{\imath \sigma_{0(0)}\left(\sigma_{0(0)}-2\right)}{2 m+1} w r T_{m m}^{(0)}(r)$,

$\Delta \mu_{\mathrm{e} m+1, m}^{1(0)}(r)=\frac{\imath \sigma_{0(0)}\left(\sigma_{0(0)}-2\right)}{2 m+1} \frac{h}{c^{2} n_{\mathrm{e}}} r T_{z m m}^{(0)}(r)$. 
After substituting the expressions for $\delta P^{1(0)}$ and $\Delta \mu_{\mathrm{e}}^{1(0)}$ into equations (11) and (14), one can finally obtain the solution for $T_{z m m}^{(0)}(r)$ and $T_{m m}^{(0)}(r)$ (see Kantor \& Gusakov 2017, Appendix B),

$$
\begin{aligned}
& T_{z m m}^{(0)}(r)=C_{1} \frac{n_{\mathrm{e}}(r)}{h(r)} r^{m}, \\
& T_{m m}^{(0)}(r)=r^{m}\left(C_{0}+C_{1} \int_{0}^{r} \frac{\mu_{\mathrm{n}}\left(r_{1}\right)}{c^{4} w^{2}\left(r_{1}\right)} \frac{d P\left(r_{1}\right)}{d r_{1}} \frac{\partial n_{\mathrm{b}}}{\partial \Delta \mu_{\mathrm{e}}}\left(r_{1}\right) \mathrm{d} r_{1}\right) .
\end{aligned}
$$

The integration constants $C_{0}$ and $C_{1}$ have to be determined from the first-order equations.

\subsection{First-order solution}

To find the eigenfrequency correction $\sigma_{0(1)}$ and the constants $C_{0}$ and $C_{1}$, it is sufficient to consider only the continuity equations (9)-(10) as well as $\theta$-components of the Euler equation (12) and the superfluid equation (15).

$\theta$-component of the Euler equation reads, in first order in $\Delta h$ [i.e. ignoring quadratically small terms like $\left.\sigma_{0(1)} \xi_{\mathrm{b} \theta}^{0(1)}\right]$,

$-\sigma_{0(1)} \xi_{\mathrm{b} \theta}^{0(0)}-\sigma_{0(0)} \xi_{\mathrm{b} \theta}^{0(1)}-2 \imath \cos \theta \xi_{\mathrm{b} \phi}^{0(1)}=\frac{1}{\imath m} \frac{\partial}{\partial \theta} \sin \theta\left[-\sigma_{0(1)} \xi_{\mathrm{b} \phi}^{0(0)}-\sigma_{0(0)} \xi_{\mathrm{b} \phi}^{0(1)}+2 \imath\left(\xi_{\mathrm{b} r}^{0(1)} \sin \theta+\xi_{\mathrm{b} \theta}^{0(1)} \cos \theta\right)\right]$.

Substituting relations (17), (19), (21), (23) into equation (46) divided by $\sin \theta$ and equating coefficients at the terms proportional to $P_{m}^{m}$, one can express $Q_{m+1, m}^{(1)}(r)$ through $\xi_{\mathrm{b} r m+1, m}^{0(1)}(r)$ and $T_{m m}^{(0)}(r)$.

Similarly, using $\theta$-component of the superfluid equation,

$$
\begin{aligned}
-\sigma_{0(1)} z_{\theta}^{0(0)}-\sigma_{0(0)} z_{\theta}^{0(1)}-2 \imath \Delta & h \cos \theta z_{\phi}^{0(0)}-2 \imath \cos \theta z_{\phi}^{0(1)} \\
= & \frac{1}{\imath m} \frac{\partial}{\partial \theta} \sin \theta\left[-\sigma_{0(1)} z_{\phi}^{0(0)}-\sigma_{0(0)} z_{\phi}^{0(1)}+2 \imath\left(z_{r}^{0(1)} \sin \theta+z_{\theta}^{0(1)} \cos \theta\right)+2 \imath \Delta h z_{\theta}^{0(0)} \cos \theta\right]
\end{aligned}
$$

one can obtain an algebraic relation between $Q_{z m+1, m}^{(1)}(r), z_{r m+1, m}^{0(1)}(r)$ and $T_{z m m}^{(0)}(r)$.

Now, taking the coefficient at $P_{m+1}^{m}$ in the continuity equation for baryons (9)

$\frac{1}{n_{\mathrm{b}}} \frac{1}{r^{2}} \frac{\partial}{\partial r} r^{2} n_{\mathrm{b}} \xi_{\mathrm{b} r}^{0(1)}+\frac{1}{r \sin \theta}\left[\frac{\partial}{\partial \theta} \sin \theta \frac{\partial Q^{(1)}}{\partial \theta}-\frac{m^{2} Q^{(1)}}{\sin \theta}\right]=0$

expressing $Q_{m+1, m}^{(1)}$ through $T_{m m}^{(0)}$ and $\xi_{\mathrm{b} r, m+1, m}^{(1)}$, and substituting expression for $T_{m m}^{(0)}(45)$, we get a first-order inhomogeneous ODE for $\xi_{\mathrm{b} r, m+1, m}$ :

$\frac{d}{d r} \xi_{\mathrm{b} r, m+1, m}^{0(1)}+A(r) \xi_{\mathrm{b} r, m+1, m}^{0(1)}-\sigma_{0(1)} C_{0} B_{10}(r)-\sigma_{0(1)} C_{1} B_{11}(r)=0$,

where $A(r), B_{10}(r), B_{11}(r)$ are known functions of $r$. The solution to this equation is

$\xi_{\mathrm{b} r, m+1, m}^{0(1)}(r)=H(r)\left[\xi_{0}+\sigma_{0(1)} C_{0} \int_{0}^{r} \frac{B_{10}(x)}{H(x)} \mathrm{d} x+\sigma_{0(1)} C_{1} \int_{0}^{r} \frac{B_{11}(x)}{H(x)} \mathrm{d} x\right], \quad H(r) \equiv \exp \left(-\int A(r) \mathrm{d} r\right)=\frac{1}{n_{\mathrm{b}}(r) r^{m+3}}$.

Since $\xi_{\mathrm{b} r, m+1, m}^{0(1)}(r)$ should be finite at $r \rightarrow 0$ the integration constant $\xi_{0}=0$.

Following the same procedure for electron continuity equation (10), we obtain the expression for $z_{r, m+1, m}^{0(1)}(r)$,

$z_{r, m+1, m}^{0(1)}(r)=H_{z}(r)\left[z_{0}+C_{1} \int_{r_{\mathrm{sfl} 1}}^{r} \frac{B_{z 01}(x)}{H_{z}(x)} \mathrm{d} x+\sigma_{0(1)} C_{0} \int_{r_{\mathrm{sfl} 11}}^{r} \frac{B_{z 10}(x)}{H_{z}(x)} \mathrm{d} x+\sigma_{0(1)} C_{1} \int_{r_{\mathrm{sfl} 11}}^{r} \frac{B_{z 11}(x)}{H_{z}(x)} \mathrm{d} x\right]$,

$H_{z}(r) \equiv \exp \left(-\int A_{z}(r) \mathrm{d} r\right)=\frac{1}{n_{\mathrm{e}}(r) r^{m+3}}$,

where $A_{z}(r), B_{z 01}(r), B_{z 10}(r), B_{z 11}(r)$ are known functions of $r$, and $r_{\mathrm{sfl} 1}$ is the inner boundary of superfluid region. If $r_{\mathrm{sfl} 1}=0$, then the integration constant $z_{0}=0$, because $z_{r}$ should be finite at $r=0$; otherwise $z_{0}$ is still zero because of the boundary condition $z_{r}=0$ at the boundary of superfluid region.

The finiteness of $\xi_{\mathrm{b} r}$ at the stellar surface $r=R$ and vanishing of $z_{r}$ at the outer superfluid boundary $r=r_{\mathrm{sfl} 2}$ imply

$\sigma_{0(1)} C_{0} \int_{0}^{R} \frac{B_{10}(x)}{H(x)} \mathrm{d} x+\sigma_{0(1)} C_{1} \int_{0}^{R} \frac{B_{11}(x)}{H(x)} \mathrm{d} x=0$,

$C_{1} \int_{r_{\mathrm{sfl} 11}}^{r_{\mathrm{sfl} 2}} \frac{B_{z 01}(x)}{H_{z}(x)} \mathrm{d} x+\sigma_{0(1)} C_{0} \int_{r_{\mathrm{sfl} 1}}^{r_{\mathrm{sfl} 12}} \frac{B_{z 10}(x)}{H_{z}(x)} \mathrm{d} x+\sigma_{0(1)} C_{1} \int_{r_{\mathrm{sfl} 1}}^{r_{\mathrm{sfl} 12}} \frac{B_{z 11}(x)}{H_{z}(x)} \mathrm{d} x=0$.

The system (53)-(54) has two independent solutions. ${ }^{8}$ The first solution is $\sigma_{0(1)}=C_{1}=0$; it is the normal $r$-mode,

$\sigma_{0}=\sigma_{0(0)}=\frac{2}{m+1}, \quad T_{m m}^{(0)}=C_{0} r^{m}, \quad T_{z m m}^{(0)}=0$.

The second one, having $\sigma_{0(1)} \neq 0$ and $C_{1} \neq 0$, is the superfluid $r$-mode.

8 The constant $C_{0}$ can be set to arbitrary value (e.g. $C_{0}=1$ ) by choosing an appropriate normalization for eigenfunctions. 
We compared this analytical solution with the numerical one (see Section 4) and found that the difference between the eigenfrequencies calculated with these two approaches does not exceed $1 \%$ even at low temperatures, where $\Delta h$ is relatively large, $\Delta h \sim 0.2-0.25$.

The next possible step would be to combine two approximate methods and calculate $r$-mode eigenfrequencies accounting for both entrainment and next-to-leading-order terms in $\Omega$. Entrainment may significantly shift the resonance temperatures, where an avoided crossing of normal and superfluid $r$-mode occurs, and thus affect the shape of $r$-mode instability window.

\section{SHORT-WAVELENGTH ANALYSIS FOR INERTIAL MODES}

In this section we obtain a dispersion relation for inertial modes in superfluid npe matter and analyze it in different limiting cases in order to explain behaviour of the modes at low and high temperatures.

Let us find the dispersion relation for inertial modes defined by equations (9)-(16) in the short-wavelength limit, in which derivatives of any perturbation $\delta A$ can be replaced as $\frac{\partial}{\partial \boldsymbol{r}} \delta A \rightarrow-\imath \boldsymbol{k} \delta A$, where $\boldsymbol{k}$ is the wave vector.

Continuity equation for baryons (9) in this limit reads:

$-\imath \boldsymbol{k} \boldsymbol{\xi}_{\mathrm{b}}^{0}=0$.

Here we omitted the term $\xi_{\mathrm{b} r}^{0} \mathrm{~d}\left(\ln n_{\mathrm{b}}\right) / \mathrm{d} r$ in comparison to $-\imath \boldsymbol{k} \boldsymbol{\xi}_{\mathrm{b}}^{0}$, since the wavelength is assumed to be much smaller than the density scale height, $k^{-1} \ll\left|\mathrm{d} \ln n_{\mathrm{b}} / \mathrm{d} r\right|^{-1}$.

Subtracting continuity equation for electrons (10) from equation (9), one obtains

$-\imath \boldsymbol{k} \boldsymbol{z}^{0}-\xi_{\mathrm{b} r}^{0} \frac{d \ln x_{\mathrm{e}}}{d r}=0, \quad x_{\mathrm{e}} \equiv \frac{n_{\mathrm{e}}}{n_{\mathrm{b}}}$.

Euler equation (11)-(13) and superfluid equation (14)-(16) read:

$-\sigma_{0}^{2} \xi_{\mathrm{b} r}^{0}-2 \imath \sigma_{0} \sin \theta \xi_{\mathrm{b} \phi}^{0}=\imath k_{r} \frac{\delta P^{1}}{w}+\frac{\mu_{\mathrm{n}}}{w^{2} c^{2}} \frac{\partial n_{\mathrm{b}}}{\partial \Delta \mu_{\mathrm{e}}} \Delta \mu_{\mathrm{e}}^{1} \frac{d P}{d r}$

$-\sigma_{0}^{2} \xi_{\mathrm{b} \theta}^{0}-2 \imath \sigma_{0} \cos \theta \xi_{\mathrm{b} \phi}^{0}=\imath k_{\theta} \frac{\delta P^{1}}{w}$,

$-\sigma_{0}^{2} \xi_{\mathrm{b} \phi}^{0}+2 \imath \sigma_{0}\left(\xi_{\mathrm{b} r}^{0} \sin \theta+\xi_{\mathrm{b} \theta}^{0} \cos \theta\right)=\imath k_{\phi} \frac{\delta P^{1}}{w}$,

$-\sigma_{0}^{2} z_{r}^{0}-2 \imath \frac{h_{1}}{h} \sigma_{0} \sin \theta z_{\phi}^{0}=\imath k_{r} \frac{c^{2} n_{\mathrm{e}}}{h} \Delta \mu_{\mathrm{e}}^{1}$,

$-\sigma_{0}^{2} z_{\theta}^{0}-2 \imath \sigma_{0} \frac{h_{1}}{h} \cos \theta z_{\phi}^{0}=\imath k_{\theta} \frac{c^{2} n_{\mathrm{e}}}{h} \Delta \mu_{\mathrm{e}}^{1}$,

$-\sigma_{0}^{2} z_{\phi}^{0}+2 \imath \frac{h_{1}}{h} \sigma_{0}\left(z_{r}^{0} \sin \theta+z_{\theta}^{0} \cos \theta\right)=\imath k_{\phi} \frac{c^{2} n_{\mathrm{e}}}{h} \Delta \mu_{\mathrm{e}}^{1}$.

Equations (56)-(63) can be written in a form $\mathbf{A} \cdot \boldsymbol{x}=0$, where $\boldsymbol{x}=\left(\delta P^{1}, \Delta \mu_{\mathrm{e}}^{1}, \xi_{\mathrm{b} r}^{0}, \xi_{\mathrm{b} \theta}^{0}, \xi_{\mathrm{b} \phi}^{0}, z_{r}^{0}, z_{\theta}^{0}, z_{\phi}^{0}\right)$, and $\mathbf{A}$ is a $8 \times 8$ matrix. Dispersion relation between the frequency $\sigma=\sigma_{0} \Omega$ and the wave vector $\boldsymbol{k}$ can be found by solving the equation $\operatorname{det} \mathbf{A}=0$, which reduces to a biquadratic equation in $\sigma_{0}$,

$A \sigma_{0}^{4}+B \sigma_{0}^{2}+C=0$,

where the coefficients $A, B, C$ are defined as

$A=k^{4}-\frac{y}{n_{\mathrm{e}}^{2}} \frac{\partial n_{\mathrm{b}}}{\partial \Delta \mu_{\mathrm{e}}} \frac{d P}{d r} \frac{d x_{\mathrm{e}}}{d r}\left(k^{2}-k_{r}^{2}\right)$,

$B=-4 k^{2} \frac{(\boldsymbol{\Omega} \boldsymbol{k})^{2}}{\Omega^{2}}\left[\left(\frac{h_{1}}{h}\right)^{2}+1\right]+4\left(\frac{h_{1}}{h}\right)^{2} \frac{y}{n_{\mathrm{e}}^{2}} \frac{\partial n_{\mathrm{b}}}{\partial \Delta \mu_{\mathrm{e}}} \frac{d P}{d r} \frac{d x_{\mathrm{e}}}{d r}\left(k^{2}-k_{r}^{2}\right)$,

$C=16\left(\frac{h_{1}}{h}\right)^{2} \frac{(\boldsymbol{\Omega} \boldsymbol{k})^{4}}{\Omega^{4}}$.

To get further insight into the problem, let us introduce the following quantities: equilibrium speed of sound $c_{\mathrm{eq}}$, adiabatic speed of sound $c_{\mathrm{fr}}$ and the coupling parameter $s$, which are defined as

$c_{\mathrm{eq}}^{2} \equiv c^{2} \frac{\mathrm{d} P / \mathrm{d} r}{\mu_{\mathrm{n}} \mathrm{d} n_{\mathrm{b}} / \mathrm{d} r}, \quad c_{\mathrm{fr}}^{2} \equiv c^{2} \frac{1}{\mu_{\mathrm{n}}}\left(\frac{\partial P}{\partial n_{\mathrm{b}}}\right)_{x_{\mathrm{e}}}, \quad s \equiv \frac{n_{\mathrm{e}}\left(\partial P / \partial n_{\mathrm{e}}\right)_{n_{\mathrm{b}}}}{n_{\mathrm{b}}\left(\partial P / \partial n_{\mathrm{b}}\right)_{x_{\mathrm{e}}}}$.

The derivative $\frac{\partial n_{\mathrm{b}}}{\partial \Delta \mu_{\mathrm{e}}}$ can be expressed in terms of these variables as

$\left(\frac{\partial n_{\mathrm{b}}}{\partial \Delta \mu_{\mathrm{e}}}\right)_{P}=\frac{n_{\mathrm{b}} n_{\mathrm{e}}}{s w}\left(\frac{1}{c_{\mathrm{eq}}^{2}}-\frac{1}{c_{\mathrm{fr}}^{2}}\right)$. 
Also, using the hydrostatic equilibrium condition, we are able to express gradients of equilibrium quantities $\left(P\right.$ and $\left.x_{\mathrm{e}}\right)$ through gravitational acceleration $g$,

$\frac{d P}{d r}=-w g, \quad \frac{d x_{\mathrm{e}}}{d r}=g \frac{x_{e}}{s}\left(\frac{1}{c_{\mathrm{eq}}^{2}}-\frac{1}{c_{\mathrm{fr}}^{2}}\right)$.

Further, let us introduce the Brunt-Väisälä frequency $\mathcal{N} \equiv g\left(1 / c_{\mathrm{eq}}^{2}-1 / c_{\mathrm{fr}}^{2}\right)^{1 / 2}$, which enters the dispersion relation for $g$-modes (Reisenegger \& Goldreich 1992), and the 'superfluid' speed $c_{\mathrm{SFL}} \equiv s c_{\mathrm{eq}} c_{\mathrm{fr}} / \sqrt{y\left(c_{\mathrm{fr}}^{2}-c_{\mathrm{eq}}^{2}\right)}$. The latter quantity in the limit $y \rightarrow \infty$ (or, equivalently, $T \rightarrow T_{\mathrm{cn}}$ ), equals to the superfluid speed of sound (see e.g. Andersson \& Comer 2001; Gusakov \& Andersson 2006).

Using the above definitions, the coefficients $A$ and $B$ from equations (65)-(66) can be rewritten in the form

$A=k^{4}+\frac{\mathcal{N}^{2}\left(k^{2}-k_{r}^{2}\right)}{c_{\mathrm{SFL}}^{2}}$,

$B=-4 k^{2} \frac{(\boldsymbol{\Omega} \boldsymbol{k})^{2}}{\Omega^{2}}\left[\left(\frac{h_{1}}{h}\right)^{2}+1\right]-4\left(\frac{h_{1}}{h}\right)^{2} \frac{\mathcal{N}^{2}\left(k^{2}-k_{r}^{2}\right)}{c_{\mathrm{SFL}}^{2}}$.

Now, if we substitute $A, B$ and $C$ into a dispersion relation $\sigma^{2}=\Omega^{2}\left(-B \pm \sqrt{B^{2}-4 A C}\right) /(2 A)$, the result will be rather lengthy. To make it more clear, let us note that at low temperatures the ratio $\mathcal{N} /\left(c_{\mathrm{SFL}} k\right)$ is $\operatorname{small,} \mathcal{N} /\left(c_{\mathrm{SFL}} k\right) \ll 1$. For example, for a wavenumber $k=10^{-5} \mathrm{~cm}^{-1}$ and a NS model used in Section 4 at a distance from the center $r=R / 2$ and temperature $T=10^{7} \mathrm{~K}$ this ratio equals $\mathcal{N} /\left(c_{\mathrm{SFL}} k\right)=0.013 .{ }^{9}$

In this limit the dispersion relation has the following form,

$$
\begin{aligned}
\sigma^{2} & =4 \frac{(\boldsymbol{\Omega} \boldsymbol{k})^{2}}{k^{2}}-4 \frac{\mathcal{N}^{2}}{c_{\mathrm{SFL}}^{2} k^{2}} \frac{\left(k^{2}-k_{r}^{2}\right)}{k^{4}} \frac{\left(\frac{h_{1}}{h}\right)^{2} \Omega^{2} k^{2}-(\boldsymbol{\Omega} \boldsymbol{k})^{2}}{\left(\frac{h_{1}}{h}\right)^{2}-1}+O\left(\frac{\mathcal{N}^{4}}{c_{\mathrm{SFL}}^{4} k^{4}}\right), \\
\sigma^{2} & =4\left(\frac{h_{1}}{h}\right)^{2} \frac{(\boldsymbol{\Omega} \boldsymbol{k})^{2}}{k^{2}}+4 \frac{\mathcal{N}^{2}}{c_{\mathrm{SFL}}^{2} k^{2}} \frac{\left(k^{2}-k_{r}^{2}\right)}{k^{4}}\left(\frac{h_{1}}{h}\right)^{4} \frac{\Omega^{2} k^{2}-(\boldsymbol{\Omega} \boldsymbol{k})^{2}}{\left(\frac{h_{1}}{h}\right)^{2}-1}+O\left(\frac{\mathcal{N}^{4}}{c_{\mathrm{SFL}}^{4} k^{4}}\right) .
\end{aligned}
$$

The first relation (73) describes normal $i^{o}$-modes. In barotropic matter, where $\mathcal{N}=0$, they have a standard dispersion relation, $\sigma^{2}=4(\boldsymbol{\Omega} \boldsymbol{k})^{2} / k^{2}$ (Landau \& Lifshitz 1987). The second relation (74) describes superfluid $i^{s}$-modes, for which the leading term differs from that of $i^{o}$-modes by the factor $\left(h_{1} / h\right)^{2}$. One can conclude that, as the entrainment effect decreases, for a given superfluid $i^{s}$-mode, its frequency approaches the frequency of its normal $\left(i^{o}\right.$-mode) counterpart. Indeed, we observe such behaviour for $i^{s}$-modes in Fig. 4 at $T<T_{\mathrm{cp}}$. Note, however, that the relations (73) and (74) are invalid if there is no entrainment at all $\left(Y_{\mathrm{np}}=0\right.$ and therefore $\left.h_{1}=h\right)$, since they contain terms that are proportional to $\left[\left(h_{1} / h\right)^{2}-1\right]^{-1}$. The asymptotic expansion for the case $h_{1}=h$ reads

$\sigma^{2}=4 \frac{(\boldsymbol{\Omega} \boldsymbol{k})^{2}}{k^{2}} \pm 4 \frac{\mathcal{N}}{c_{\mathrm{SFL}} k} \frac{\sqrt{\left(k^{2}-k_{r}^{2}\right)}}{k^{3}}|\boldsymbol{\Omega} \boldsymbol{k}| \sqrt{\Omega^{2} k^{2}-(\boldsymbol{\Omega} \boldsymbol{k})^{2}}+O\left(\frac{\mathcal{N}^{2}}{c_{\mathrm{SFL}}^{2} k^{2}}\right)$

where the ' + ' sign refers to $i^{s}$-mode, and the ' - ' sign refers to the $i^{o}$-mode. The frequencies of normal and superfluid $i$-modes coincide up to the terms proportional to $\mathcal{N} /\left(c_{\mathrm{SFL}} k\right) \ll 1$; indeed, one can see in Fig. 2, that the frequencies of $l_{0}-m=3$ and $l_{0}-m=5 i^{s}$-modes in the case $Y_{\mathrm{np}}=0$ are very close to the corresponding $i^{o}$-modes at low temperatures $T \ll T_{\mathrm{cn}}$. Fig. 4 also illustrates this point: when protons become non-superfluid (at $T>T_{\mathrm{cp}}$ ), the entrainment effect vanishes and, while the temperature is still much less than $T_{\mathrm{cn}}, i^{s}$-modes and $i^{\circ}$-modes with the same $l_{0}$ are close to each other.

Now let us examine the behaviour of inertial modes in the limit of high temperatures $T \rightarrow T_{\mathrm{cn}}$. Since the quantity $y$ in this limit tends to infinity, $y \rightarrow \infty$, the 'superfluid' speed tends to zero, $c_{\mathrm{SFL}} \rightarrow 0$. The Brunt-Väisälä frequency, on the other hand, does not depend on temperature. Thus, the ratio $\mathcal{N} /\left(c_{\mathrm{SFL}} k\right)$ can approach arbitrarily large values, and asymptotic expansions (73)-(75) are invalid. In this case one should consider the opposite limit, $c_{\mathrm{SFL}} k / \mathcal{N} \ll 1$, in which the asymptotic expansion for the dispersion relation reads

$\sigma^{2}=4 \frac{c_{\mathrm{SFL}}^{2} k^{2}}{\mathcal{N}^{2}} \frac{(\boldsymbol{\Omega} \boldsymbol{k})^{4}}{\Omega^{2} k^{2}\left(k^{2}-k_{r}^{2}\right)}+O\left(\frac{c_{\mathrm{SFL}}^{4} k^{4}}{\mathcal{N}^{4}}\right)$

$\sigma^{2}=4\left(\frac{h_{1}}{h}\right)^{2} \Omega^{2}-4 \frac{c_{\mathrm{SFL}}^{2} k^{2}}{\mathcal{N}^{2}} \frac{\left[\Omega^{2} k^{2}-(\boldsymbol{\Omega} \boldsymbol{k})^{2}\right]\left[\left(\frac{h_{1}}{h}\right)^{2} \Omega^{2} k^{2}-(\boldsymbol{\Omega} \boldsymbol{k})^{2}\right]}{\Omega^{2} k^{2}\left(k^{2}-k_{r}^{2}\right)}+O\left(\frac{c_{\mathrm{SFL}}^{4} k^{4}}{\mathcal{N}^{4}}\right)$.

Here we see that the normal and superfluid modes, described by equations (76) and (77) respectively, exhibit qualitatively

9 We remind the reader that the wavenumber $k$ is assumed to be large; in particular, it is much greater than the inverse stellar radius, $k \gg 1 / R=8.2 \times 10^{-7} \mathrm{~cm}^{-1}$. 
different behaviour as $T \rightarrow T_{\mathrm{cn}}: i^{o}$-mode frequencies vanish ${ }^{10}$, whereas $i^{s}$-mode frequencies are finite. One can clearly observe such behaviour in Fig. 2 and Fig. 4.

To sum up, in this section we obtained dispersion relations for normal and superfluid inertial modes in superfluid npe matter in a short wavelength limit. We analyzed this relations in two opposite limiting cases. The first case, $c_{\mathrm{SFL}} k / \mathcal{N} \gg 1$, describes behaviour of inertial modes at low temperatures $T \ll T_{\mathrm{cn}}$. The corresponding relations are presented by equations (73)-(74) if $h_{1} \neq h$ and by equation (75) if $h_{1}=h$. These relations explain why the frequencies of superfluid $i^{s}$-modes are close to frequencies of the corresponding normal $i^{\circ}$-modes if the entrainment effect is small or absent. The second case, $c_{\mathrm{SFL}} k / \mathcal{N} \ll 1$, corresponds to the limit $T \rightarrow T_{\mathrm{cn}}$, in which $i^{\circ}$-mode frequencies (equation 76 ) tend to zero, while $i^{s}$-mode frequencies (equation 77) remain finite. These conclusions are consistent with the properties of the inertial modes spectrum calculated in Section 4 (see Fig. 2 and Fig. 4).

\section{SUMMARY}

In this paper we studied the properties of inertial modes in slowly rotating superfluid npe NSs. We calculated the spectrum of $l_{0}-|m|=1, l_{0}-|m|=3$, and $l_{0}-|m|=5$ inertial modes for $m=2$, working in the leading order in rotation and including both the entrainment and finite temperatures effects for the first time. In Section 4 we present the first results of such calculations. We worked in the Cowling approximation, in the Newtonian limit (but employed relativistic EOS) and assumed constant baryon critical temperatures throughout the core.

One of the motivations for doing this work was to find possible avoided crossings in the plane 'mode frequency - stellar temperature' between the normal $r^{o}$-mode and superfluid inertial modes ( $i^{s}$-modes). At stellar temperatures corresponding to avoided crossings, eigenfunctions of $r^{o}$-mode and $i^{s}$-mode mix with each other. This stabilizes the $r^{o}$-mode and explains the existence of hot and rapidly rotating NSs in LMXBs (Gusakov et al. 2014a,b). In our simplified physical model we did not reveal any avoided crossing of $r^{o}$-mode with the superfluid modes in the leading order in the rotation frequency $\Omega$. However, we found that at temperatures close to the critical neutron temperature one should expect stabilizing interaction of $r^{\circ}$-mode with the superfluid $r^{s}$-mode (i.e., $i^{s}$-mode with $l_{0}-|m|=1$ ). Moreover, we showed, for our particular stellar model, that at low enough temperatures $l_{0}-|m|=3 i^{s}$-mode has a frequency sufficiently close to that of $r^{o}$-mode and hence may also stabilize $r^{o}$-mode.

We also developed an approximate method which simplifies calculations of the $r^{s}$-mode eigenfrequency. This method is similar to that used by Kantor \& Gusakov (2017) and it allows one to calculate $r^{s}$-mode analytically in the limit of small entrainment (see Section 5), assuming npe core composition and working in the leading order in $\Omega$. We found a good agreement of our analytical and numerical results. In addition, in Section 6 we derived and discussed dispersion relations for normal and superfluid inertial modes in the short wavelength limit. Using these relations we explained some properties of inertial modes at low $\left(T \ll T_{\mathrm{cn}}\right)$ and high $\left(T \rightarrow T_{\mathrm{cn}}\right)$ stellar temperatures, found numerically in Section 4 .

In the future we plan to combine two analytical methods for calculating $r$-modes (the method presented in Section 5 and the method by Kantor \& Gusakov 2017), accounting for both entrainment effect and next-to-leading order corrections in $\Omega$. This will allow us to determine avoided crossings between the normal and superfluid $r$-modes (and thus determine the $r$-mode instability windows) under more realistic assumptions. We also plan to calculate the spectrum of inertial modes for more elaborated neutron star models allowing for muons and density-dependent profiles of nucleon critical temperatures in the stellar core.

\section{ACKNOWLEDGMENTS}

This work is partially supported by the Foundation for the Advancement of Theoretical Physics and Mathematics BASIS [Grant No. 17-12-204-1] and by RFBR [Grant No. 18-32-20170].

\section{REFERENCES}

Akmal A., Pandharipande V. R., Ravenhall D. G., 1998, Phys. Rev. C, 58, 1804

Andersson N., 1998, ApJ, 502, 708

Andersson N., Comer G. L., 2001, MNRAS, 328, 1129

Andersson N., Kokkotas K. D., 2001, International Journal of Modern Physics D, 10, 381

10 We remind the reader that we are considering only pure $i$-modes with $\sigma \propto \Omega$ at low rotation frequencies; in barotropic $(\mathcal{N}=0)$ star normal inertial modes survive at $T>T_{\mathrm{cn}}$, since they always have frequency $\sigma^{2}=4(\boldsymbol{\Omega} \boldsymbol{k})^{2} / k^{2}$, (see equations 73 and 75 with $\left.\mathcal{N}=0\right)$. In non-superfluid non-barotropic stars inertial modes (except for the single $r$-mode) do not exist, since in the limit $\Omega \rightarrow 0$ they turn into g-modes (Unno et al. 1989). Therefore it is not surprising that the frequencies of $i^{o}$-modes tend to zero at $T \rightarrow T_{\mathrm{cn}}$. 
Andersson N., Sidery T., Comer G. L., 2006, MNRAS, 368, 162

Andersson N., Glampedakis K., Haskell B., 2009, Phys. Rev. D, 79, 103009

Andreev A. F., Bashkin E. P., 1976, Soviet Journal of Experimental and Theoretical Physics, 42, 164

Friedman J. L., Morsink S. M., 1998, ApJ, 502, 714

Gusakov M. E., 2016, Phys. Rev. D, 93, 064033

Gusakov M. E., Andersson N., 2006, Mon. Not. R. Astron. Soc., 372, 1776

Gusakov M. E., Dommes V. A., 2016, Phys. Rev. D, 94, 083006

Gusakov M. E., Kantor E. M., Haensel P., 2009a, Phys. Rev. C, 79, 055806

Gusakov M. E., Kantor E. M., Haensel P., 2009b, Phys. Rev. C, 80, 015803

Gusakov M. E., Chugunov A. I., Kantor E. M., 2014a, Phys. Rev. D, 90, 063001

Gusakov M. E., Chugunov A. I., Kantor E. M., 2014b, Physical Review Letters, 112, 151101

Gusakov M. E., Haensel P., Kantor E. M., 2014c, MNRAS, 439, 318

Haskell B., Degenaar N., Ho W. C. G., 2012, MNRAS, 424, 93

Heiselberg H., Hjorth-Jensen M., 1999, ApJ, 525, L45

Ho W. C. G., Andersson N., Haskell B., 2011, Physical Review Letters, 107, 101101

Kantor E. M., Gusakov M. E., 2011, Phys. Rev. D, 83, 103008

Kantor E. M., Gusakov M. E., 2017, MNRAS, 469, 3928

Landau L. D., Lifshitz E., 1987, Fluid mechanics. Course of theoretical physics. Pergamon Press, Oxford

Lee U., Yoshida S., 2003, ApJ, 586, 403

Lindblom L., Ipser J. R., 1999, Phys. Rev. D, 59, 044009

Lindblom L., Mendell G., 2000, Phys. Rev. D, 61, 104003

Lockitch K. H., Friedman J. L., 1999, ApJ, 521, 764

Mahmoodifar S., Strohmayer T., 2013, ApJ, 773, 140

Mendell G., 1991, ApJ, 380, 515

Potekhin A. Y., Fantina A. F., Chamel N., Pearson J. M., Goriely S., 2013, A\&A, 560, A48

Prix R., Comer G. L., Andersson N., 2002, A\&A, 381, 178

Provost J., Berthomieu G., Rocca A., 1981, A\&A, 94, 126

Reisenegger A., Goldreich P., 1992, ApJ, 395, 240

Saio H., 1982, ApJ, 256, 717

Unno W., Osaki Y., Ando H., Saio H., Shibahashi H., 1989, Nonradial oscillations of stars

Yoshida S., Lee U., 2000, ApJS, 129, 353 